\title{
What was learned from the new VFE-2 experiments?
}

\author{
James M. Luckring ${ }^{*}$ \\ NASA Langley Research Center, Hampton, VA, USA \\ and \\ Dietrich Hummel ${ }^{\dagger}$ \\ Technical University Braunschweig, Braunschweig, Germany
}

\begin{abstract}
In the present paper the main results of the new experiments from VFE-2 are summarized. These include some force and moment results, surface and off-body measurements, as well as steady and fluctuating quantities. Some critical remarks are added, and an outlook for future investigations is given.
\end{abstract}

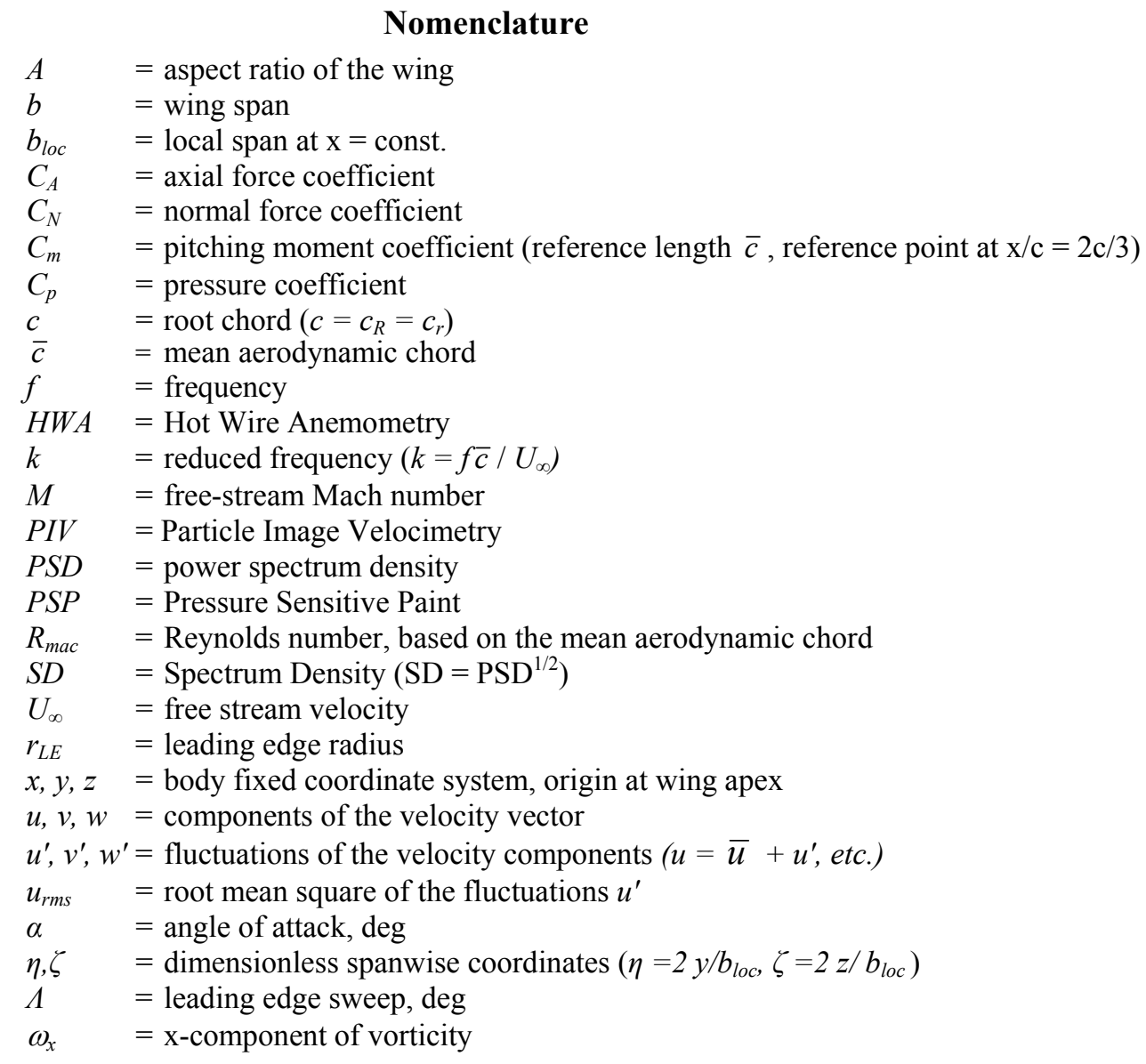

\footnotetext{
${ }^{*}$ Senior Research Engineer, NASA Langley Research Center, Hampton VA, 23681 USA, Associate Fellow AIAA.

${ }^{\dagger}$ Prof. Dr.-Ing. (retired), Institute of Fluid Mechanics, Bienroder Weg 3, 38106 Braunschweig, Germany, Associate Fellow AIAA.
} 


\section{Introduction}

$A^{t}$ the very beginning of the Second International Vortex Flow Experiment (VFE-2), comprehensive A experimental results from the National Transonic Facility (NTF) at NASA Langley Research Center on a $65^{\circ}$ swept delta wing were already available ${ }^{1}$. For one sharp and three rounded leading edges normal force and pitching moment as well as pressure distribution measurements had been carried out for a large variety of Mach numbers and Reynolds numbers. The geometry of the wing and the positions of the pressure tubes according to Ref. 1 is given in Fig. 1, and the corresponding arrangement of the wind tunnel model in the National Transonic Facility is shown in Fig. 2. The results of these measurements have been analyzed and summarized in various papers ${ }^{2-5}$.
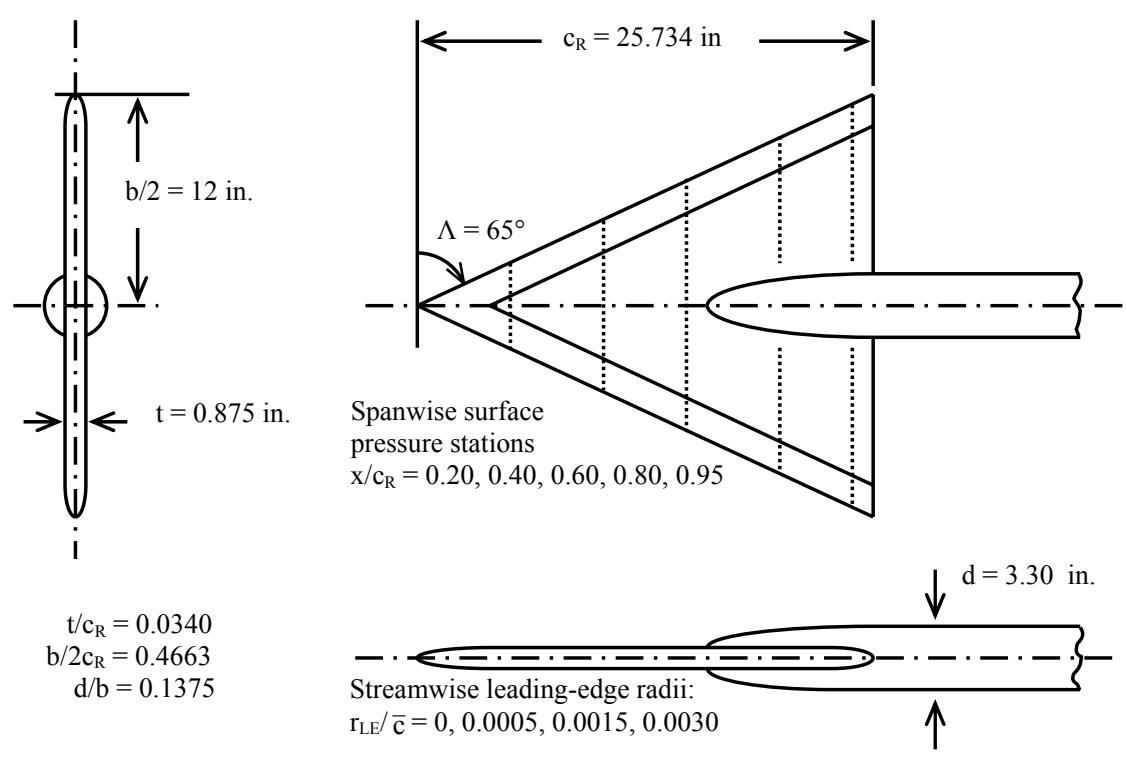

Figure 1. VFE-2 configuration: NASA delta wing $\Lambda=65^{\circ}, A=1.85$ (Ref. 1)

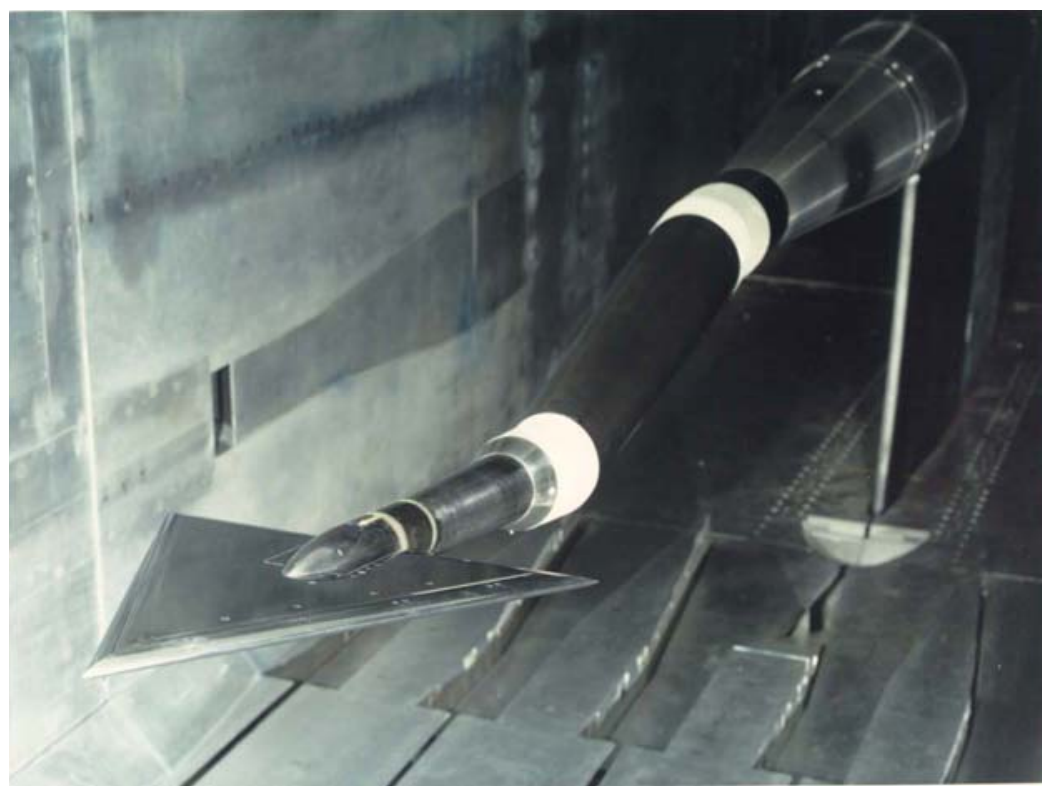

Figure 2. NASA NTF tests on the $65^{\circ}$ delta wing (Ref. 1) 
In the 2001 proposal $^{6}$ for a new vortex-flow research program, the NASA $65^{\circ}$ delta wing configuration with sharp and rounded leading edges was chosen for new tests concerning additional drag and flow field data. This led to the Second International Vortex Flow Experiment (VFE-2), which was carried out within a Research and Technology Organization (RTO) Applied Vehicle Technology (AVT) Task Group 2003 - 2007. The new experiments were aimed at an extension of the knowledge on the vortical flow around the configuration with sharp and rounded leading edges. The measurements were accompanied by numerical investigations to support the interpretation of the flow physics, and in the present paper only limited numerical details are used similarly. The history of VFE-2 has been described by D. Hummel ${ }^{7}$, and the authors ${ }^{8-12}$ of the experimental contributions to VFE-2 have presented their work in separate papers. The main results of the new experiments within VFE-2 are summarized below.

\section{Normal/axial forces and pitching moment characteristics for sharp and rounded leading edges.}

The extensive pressure instrumentation used for experiments at NASA Langley prevented the use of a conventional internal force and moment balance. However, a novel sting-gauging technique was employed to obtain normal force and pitching moment coefficient measurements over the full range of test conditions.
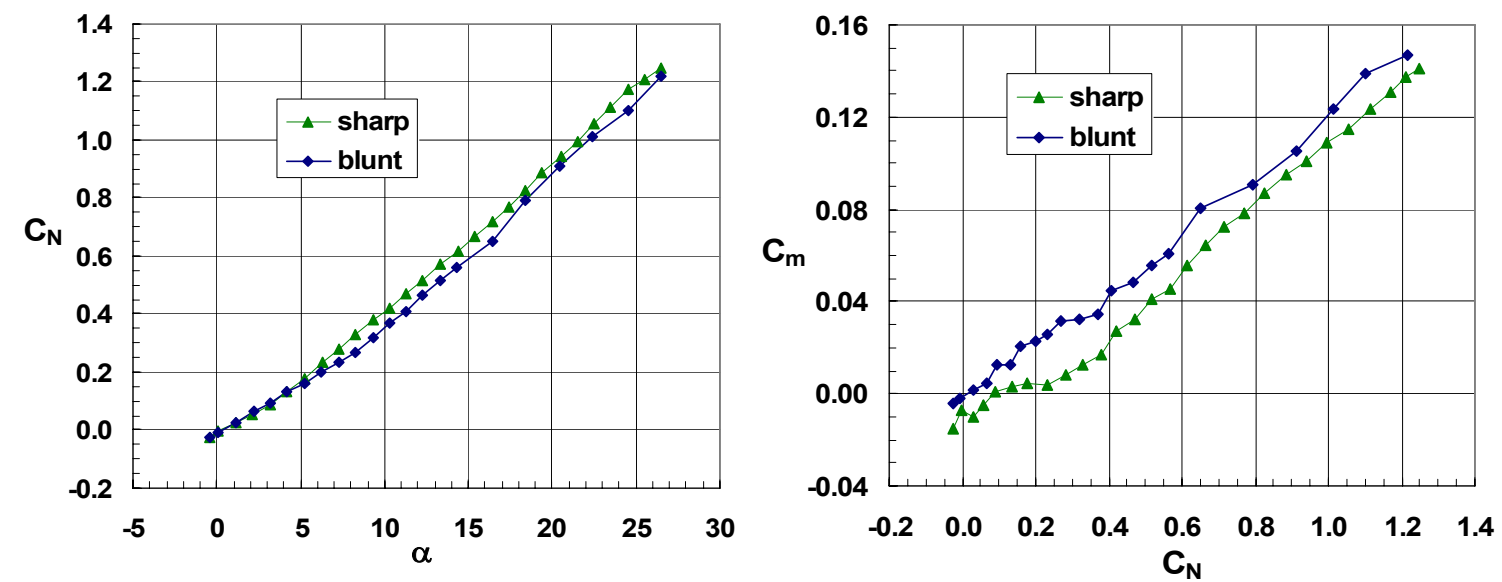

Figure 3. Effect of bluntness on experimental normal force and pitching moment coefficients for the VFE-2 configuration with sharp and medium radius rounded leading edges at $M=0.4, R_{\text {mac }}=6 \cdot 10^{6}$. Results from NASA Langley NTF.

The effect of leading-edge bluntness on the normal force and pitching moment coefficients for the VFE-2 configuration is presented in Fig. 3 from measurements taken in the NASA Langley NTF at a Mach number of 0.4 and a Reynolds number of 6 million. At low angles of attack both wings develop the same normal force. The blunt leading edge wing has attached flow at these conditions, and any leading-edge vortex separation effects for the sharp-edged wing are very small at these low angles of attack. Over the rest of the angle of attack range investigated the blunt leading edge wing develops less normal force than the sharp-edged wing. The blunt leading edge weakens the leading edge vortex ${ }^{8}$ resulting in reduced vortex-induced normal force. Pitching moments show the blunt-edged wing has a more forward center of pressure, compared to the sharp-edged wing, over the range of conditions investigated. The vortex flow physics associated with these effects may be taken from the discussions in Section III.

Low-speed compressibility effects on the normal-force coefficient are summarized in Fig. 4 with measurements taken at NASA Langley in the NTF with the full-scale delta wing and in the Low Turbulence Pressure Tunnel (LTPT) with the $3 / 4$-scale delta wing. All results are for the medium-bluntness leading edge. Although the Reynolds numbers are not identical in this comparison, the differences due to Reynolds number are considered to be small. The left-hand side of the figure shows the variation of normal-force coefficient with angle of attack for Mach numbers ranging between 0.2 and 0.6. The right-hand side shows the same data, scaled by the linear Prandtl-Glauert similarity parameter, $\beta=\left[1-\mathrm{M}^{2}\right]^{1 / 2}$. The Prandtl-Glauert scaled parameters will collapse under conditions largely governed by linear aerodynamics, and the correlation of the measurements over low to moderate angles of attack is quite good, especially considering that the results were obtained with different models in different facilities. Variations among the scaled measurements at high angles of attack are due to nonlinear aerodynamics, most 
probably due to leading-edge vortex separation. Similarity scaling of these effects requires more advanced methods than linear Prandlt-Glauert scaling.
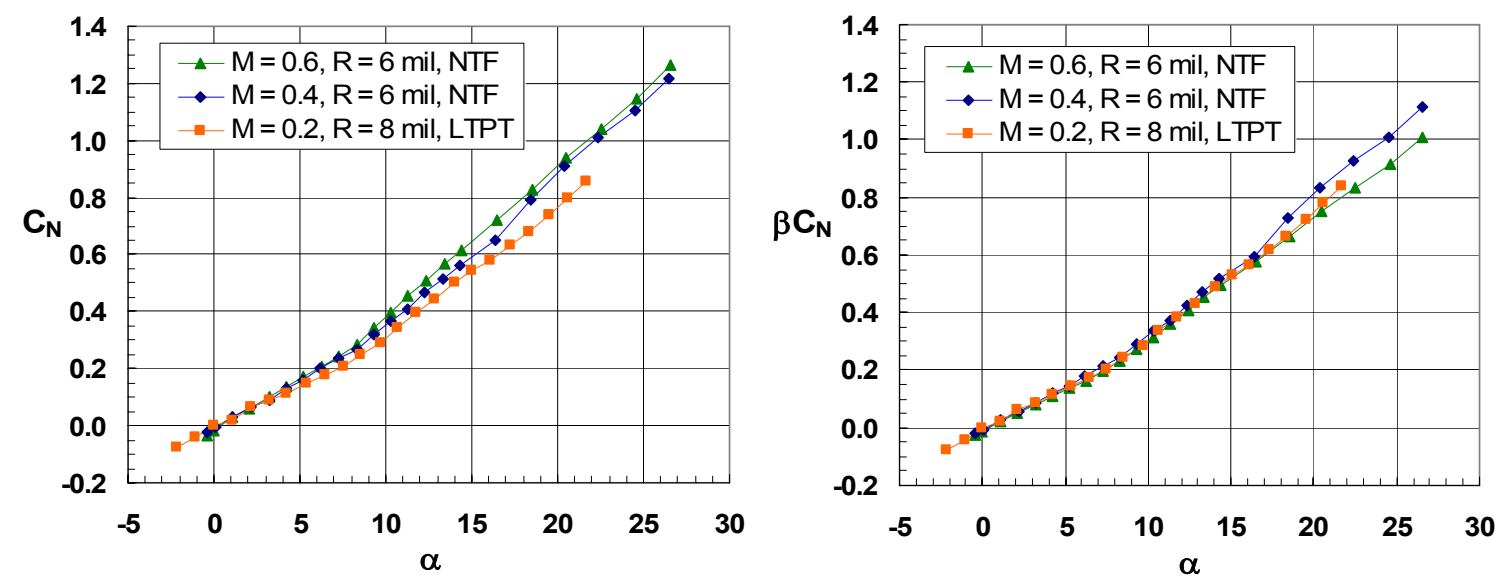

Figure 4. Effect of Mach number on experimental normal force coefficients for the VFE-2 configuration with medium radius rounded leading edge at $R_{\text {mac }}=6 \cdot 10^{6}$ or $8 \cdot 10^{6}$. Results from NASA Langley NTF and LTPT.

As mentioned earlier, the instrumentation used in the original NASA experiments did not allow for full force/moment measurement, and in particular prevented the measurement of axial forces. It would be expected that the axial force coefficient should also show a significant effect of bluntness in association with the onset and progression of leading-edge separation.
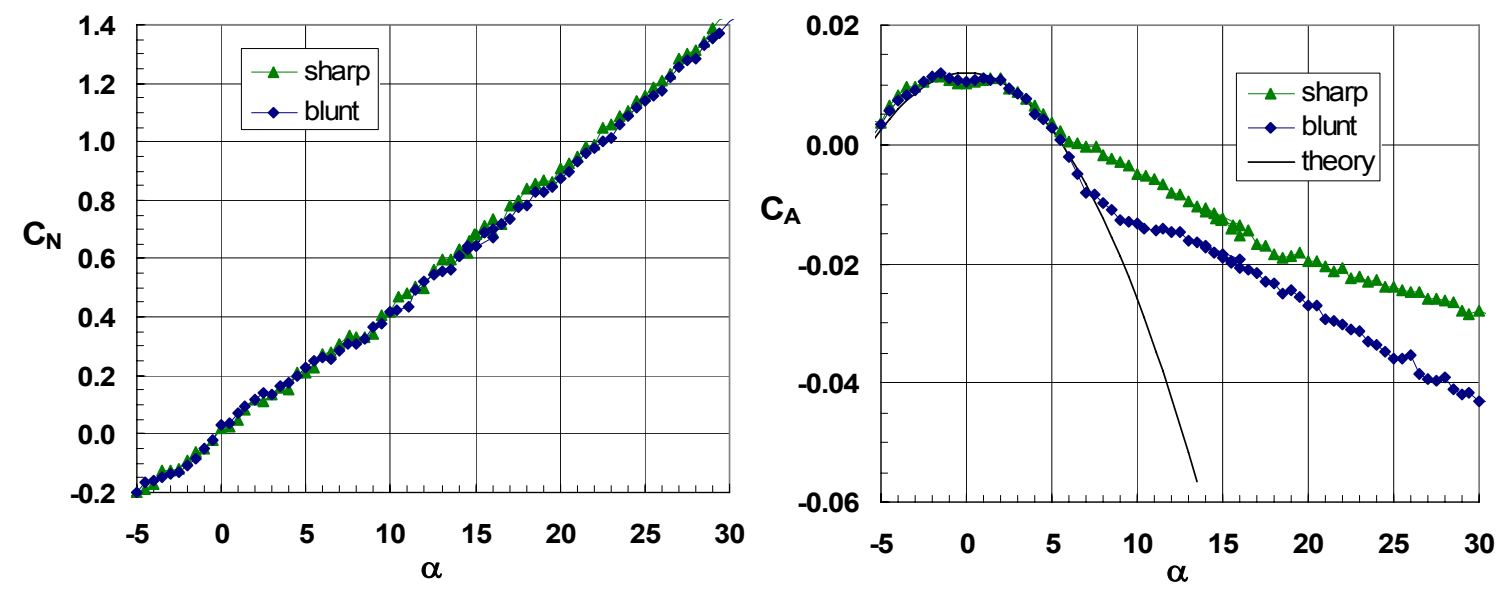

Figure 5. Effect of bluntness on experimental normal and axial force coefficients for the VFE-2 configuration with sharp and medium radius rounded leading edge at $M=0.1, R_{\mathrm{mac}}=1 \cdot 10^{6}$. Results from ONERA L1.

The effect of bluntness on axial force coefficient from recent measurements ${ }^{10}$ obtained in the ONERA L1 facility are shown in Fig. 5 for a Mach number of 0.1 and a Reynolds number of 1 million. There is some scatter in the current measurements of normal force coefficient that prevent seeing the effect due to vortex separation shown in Fig 3. However, the effect of bluntness on axial force coefficient can clearly be seen in the right-hand portion of Fig. 5. A theoretical estimate of attached flow trends is shown by fitting the data to the functional form $\mathrm{C}_{\mathrm{A}}=\mathrm{k}_{0}-\mathrm{k}_{1} \sin ^{2} \alpha$ nominally in the 3 to 6 degree angle of attack range of the blunt-edge measurements. In this equation $\mathrm{k}_{0}$ and $\mathrm{k}_{1}$ are constants determined by the fit. Bluntness resulted in an extended range of attached-flow suction, compared to the sharp edge wing, and the reduction in axial force due to bluntness was manifested over the full range of measurements obtained at ONERA. 


\section{Onset of vortical flow on the VFE-2 configuration with rounded leading edge.}

Since for delta wings with sharp leading edges and fixed primary separation many experimental investigations are available in the literature, the AVT Task Group decided in 2003 to use the case of sharp leading edges within VFE-2 as reference only and to direct the main emphasis for new experiments towards studies of the vortex formation on the configuration with rounded leading edges.

\section{A. Principal flow structure at $\alpha=13^{\circ}$.}

For the configurations with rounded leading edges the available NASA data ${ }^{1}$ showed spanwise pressure distributions with two separate suction peaks on the upper surface of the wing, see Fig. 6, which so far have never been observed for sharp edged delta wings. Therefore the AVT Task Group decided to study the onset of separated flow for rounded leading edges in more detail, and for this purpose the medium radius leading edge configuration with $r_{L E} / \bar{c}=0.0015$ has been selected.

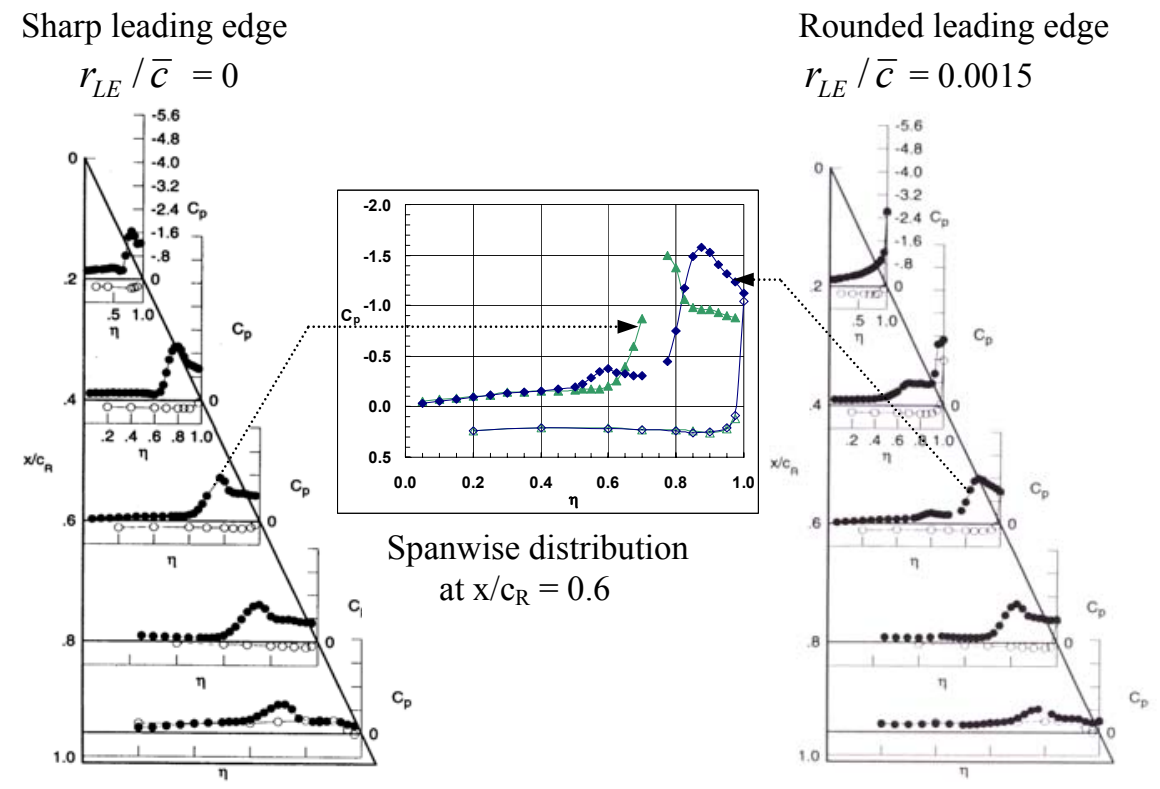

Figure 6. Effect of leading edge bluntness on the pressure distribution ${ }^{1}$ of the VFE-2 configuration at $M=0.4, R_{\operatorname{mac}}=6 \cdot 10^{6}, \alpha=13^{\circ}$, NTF data

The experimental pressure distribution on the VFE-2 configuration with medium radius rounded leading edges at Mach number $\mathrm{M}=0.4$ and Reynolds number (based on the mean aerodynamic chord) $\mathrm{R}_{\mathrm{mac}}=3$ million at an angle of attack $\alpha=13^{\circ}$ is shown in Fig. 7 resulting from the measurements at DLR Goettingen ${ }^{9,13}$. On the left-hand side the pressure distributions according to the static surface pressure (PSI) experiments for the model sections with pressure taps are shown. In general, these measurements show very similar results as the earlier NTF tests ${ }^{1}$ although precise correlation would not be expected due to the difference in Reynolds number. In the region of attached flow near the apex of the wing high suction occurs at the leading edge. Further downstream an inner suction peak develops followed by an even higher outer suction peak, which replaces the original leading edge suction. Towards the trailing edge of the wing the outer suction peak is still maintained, whereas the inner suction peak reduces more and more and finally disappears. The PSP results on the right-hand side show these features in the same way. The full view of the pressure distribution on the configuration is given in the colored figure in the centre of Fig. 7. It shows on both sides the onset of the strong outer suction peak to be located at about $\mathrm{x} / \mathrm{c}_{\mathrm{R}}=0.45$ and undoubtedly this suction peak is related to the primary vortex of the separated flow. The inner suction peak, however, could not be understood so easily. It starts earlier than the outer suction peak and the shape of its initial pressure distributions resembled that of a separation bubble related to the transition from laminar to turbulent flow ${ }^{13}$. After the formation of the primary vortex the inner suction peak reduces more and more in the downstream direction ${ }^{14,15}$. 

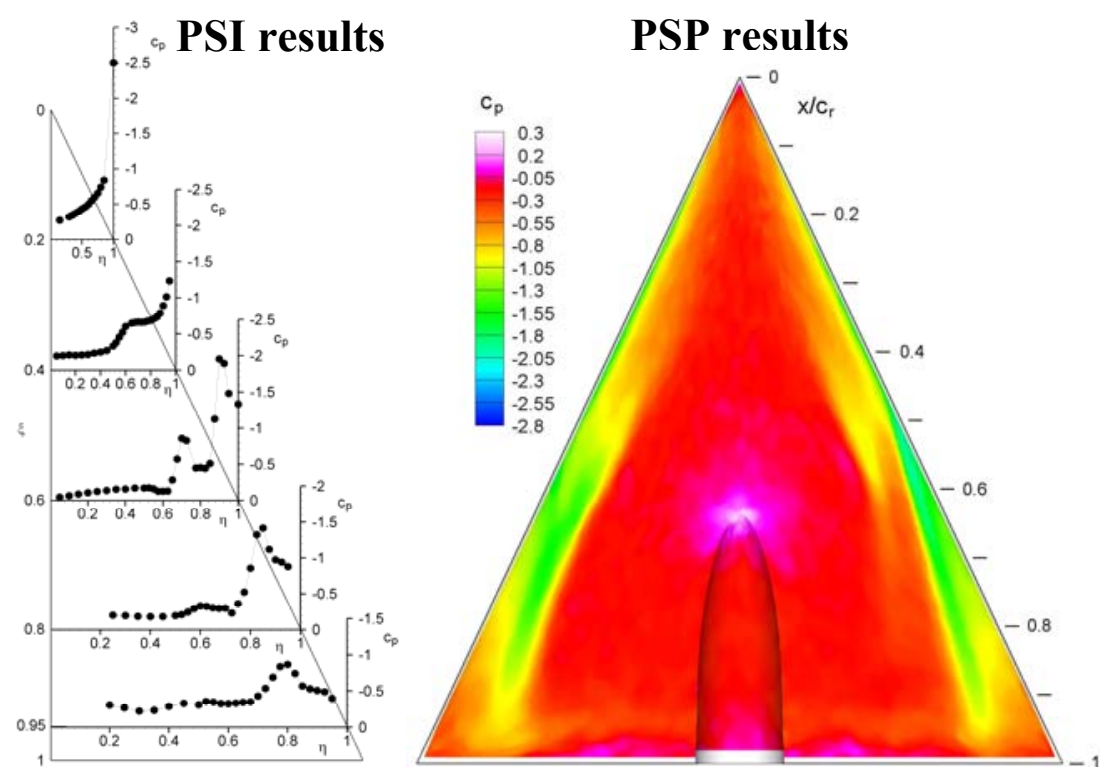

PSP results

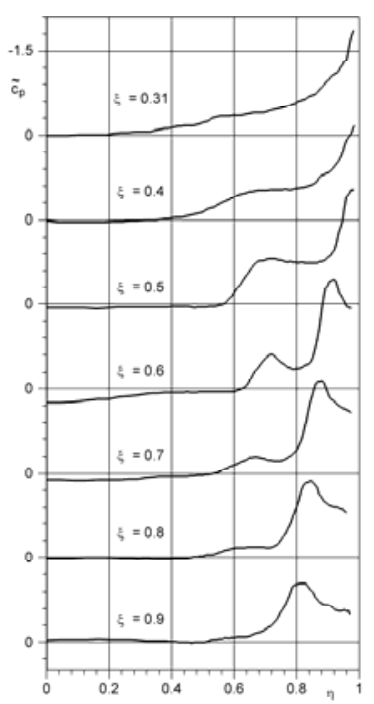

Figure 7. Experimental pressure distribution on the VFE-2 configuration with medium radius rounded leading edges for $M=0.4, R_{\mathrm{mac}}=3 \cdot 10^{6}, \alpha=13^{\circ}$. Results from DLR Goettingen

On the right-hand side of the colored central plot in Fig. 7 the inner suction region remains separate from the primary vortex, but on the left-hand side it merges into the suction area of the primary vortex. The inner and outer vortices are co-rotating, and their interaction can be sensitive to small asymmetries that arise from a variety of sources (e.g., flow angularity, model orientation, surface geometry, etc). According to detailed investigations of the model shape carried out at DLR Goettingen these asymmetries are most likely the result of imperfections of the model geometry. At this stage of the investigations the inner suction peak in the pressure distribution was thought to be the outcome of boundary layer effects resulting from a 3D bubble-type laminar/turbulent transition at about $\mathrm{x} / \mathrm{c}_{\mathrm{R}}=0.3$, and these effects could be sensitive to disturbances and to model imperfections. At about $\mathrm{x} / \mathrm{c}_{\mathrm{R}}=0.5 \mathrm{a}$ small inner vortex might have been formed, whose path downstream could be either separate or merged with the main primary vortex.

After some substantial support by numerical investigations ${ }^{16}$ within VFE-2 concerning the technical equipment $^{7}$, 3D PIV investigations ${ }^{17,18}$ have been carried out at DLR Goettingen. Fig. 8 shows the result for the flow field in various cross-sectional planes for the same free stream conditions as in Fig. 7 and in comparison with the numerical result ${ }^{16}$. The surface pressure distribution from the PSP investigations, see Fig. 7, is displayed in colors for the right half of the wing on the left side of Fig. 8. The outer suction peak underneath the primary vortex is marked by the green area and the one underneath the inner vortex can be identified by a yellow band. Due to the position of the light source in the wind tunnel there exists a certain region in the vicinity of the leading edge, which was not illuminated, and therefore no velocity measurements are available for this region. Two vortices with the same sense of rotation are clearly indicated in the rear part of the wing. Around $x / c_{r}=0.7$ the size of both vortices is about the same as predicted by the CFD results. Further downstream the outer vortex becomes the stronger one, whereas the inner vortex decays. These experimental results are in excellent qualitative agreement with the numerical findings ${ }^{16}$ as shown in the right-hand figure. The results of the two campaigns in the Transonic Wind Tunnel at Goettingen in 2004 and 2005 have been summarized ${ }^{19}$ and they were also a subject of the 2006 AIAA achievements review $^{20}$ as well as to some VFE-2 status reports ${ }^{21,22}$.

The understanding of the onset of the vortical flow on the present delta wing with rounded leading edge can be summarized as follows: On the medium radius rounded leading edge VFE-2 configuration at $\alpha=13^{\circ}$ the first flow separation takes place in the front one-third of the wing, where the wing is relatively thick. At its very beginning the separated flow region is located close to the wing surface, but further downstream a concentrated inner primary vortex is formed quite rapidly. Along the blunt leading edge the curvature radius of the leading edge remains constant, whereas the local half span increases downstream. This means that the leading edge becomes relatively sharper $\left(r_{L E} / b_{l o c}\right)$ towards the trailing edge of the wing. Correspondingly the suction at the leading edge increases 
downstream, and finally new flow separations take place in the outer portion of the rear part of the wing, forming an outer primary vortex there. In the region of the onset of this outer primary vortex, strong interference with the already existing inner primary vortex takes place. The dominant part of vorticity, shed from the leading edge, is now fed into the outer primary vortex, whereas the feeding of the inner vortex with vorticity is reduced. Therefore the inner primary vortex decays downstream due to dominating viscous effects. Unfortunately within VFE-2 not all details of the onset of vortical flow in the front part of the configuration could be investigated experimentally because of geometric constraints of the PIV setup in the wind tunnel, but due to the encouraging agreement between the experimental and the calculated results, further study of these details can be guided with analysis of numerical solutions.

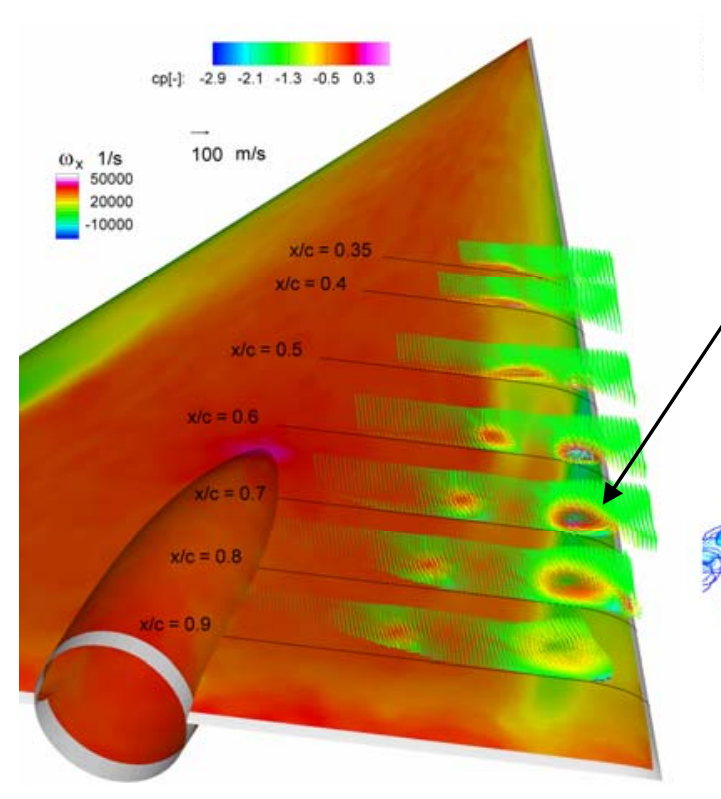

Experiment

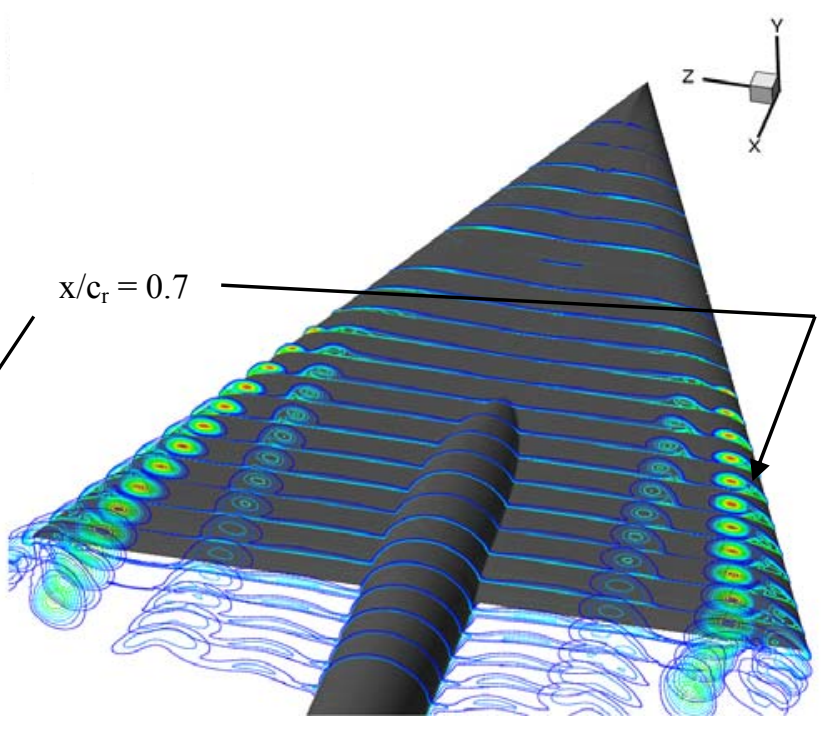

Numerical solution

Figure 8. Pressure (surface color), velocity (vectors) and vorticity (vector color) distributions above the VFE-2 configuration with rounded leading edges (medium radius) for $M=0.4, R_{\operatorname{mac}}=3 \cdot 10^{6}, \alpha=13^{\circ}$. Comparison of the PSP and PIV measurements ${ }^{19}$ at DLR Goettingen (left) with the numerical solution ${ }^{16}$ of EADS Munich (right).

\section{B. Variation of the angle of attack}

The flow pattern described so far depends on the angle of attack. From previous studies ${ }^{2-5}$ the progression of leading-edge flow separation of the outer primary vortex with angle of attack was established as (i) a low angle-ofattack range where the flow was attached, (ii) an intermediate angle-of-attack range where the primary separation progressed up the leading edge, from trailing edge to apex, with increasing angle of attack, and (iii) a high angle-ofattack range where essentially the entire leading edge exhibited primary vortex separation.

A series of pressure distributions for $\mathrm{M}=0.4$ and $\mathrm{R}_{\mathrm{mac}}=3$ million and for various angles of attack from the PSP measurements ${ }^{15,19}$ at DLR Goettingen is shown in Fig. 9. Up to $\alpha=11.2^{\circ}$ only the inner primary vortex exists, and the corresponding suction on the wing surface reaches considerable values near the trailing edge. With further increasing angle of attack the outer primary vortex is formed in the rear part of the configuration, and already at $\alpha=$ $12.2^{\circ}$ its onset has reached a position at $\mathrm{x} / \mathrm{c}_{\mathrm{R}}=0.6$. The strength of the inner primary vortex increases up to the region of the onset of the outer primary vortex, but then decreases suddenly downstream towards the trailing edge. This is due to the fact that the vorticity shed from the leading edge is now fed into the outer primary vortex, and this leads to the considerable reduction of the strength of the inner primary vortex. Another effect can also be recognized from Fig. 9: In that region where an outer primary vortex already exists, the weakened inner primary vortex moves inboard. For further increasing angles of attack the onset of the outer primary vortex moves upstream, see $\alpha=13.3^{\circ}$ and $\alpha=15.3^{\circ}$. At $\alpha=20.5^{\circ}$ the outer primary vortex covers almost the whole leading edge, but some weak remnants of an inner primary vortex can still be detected. 

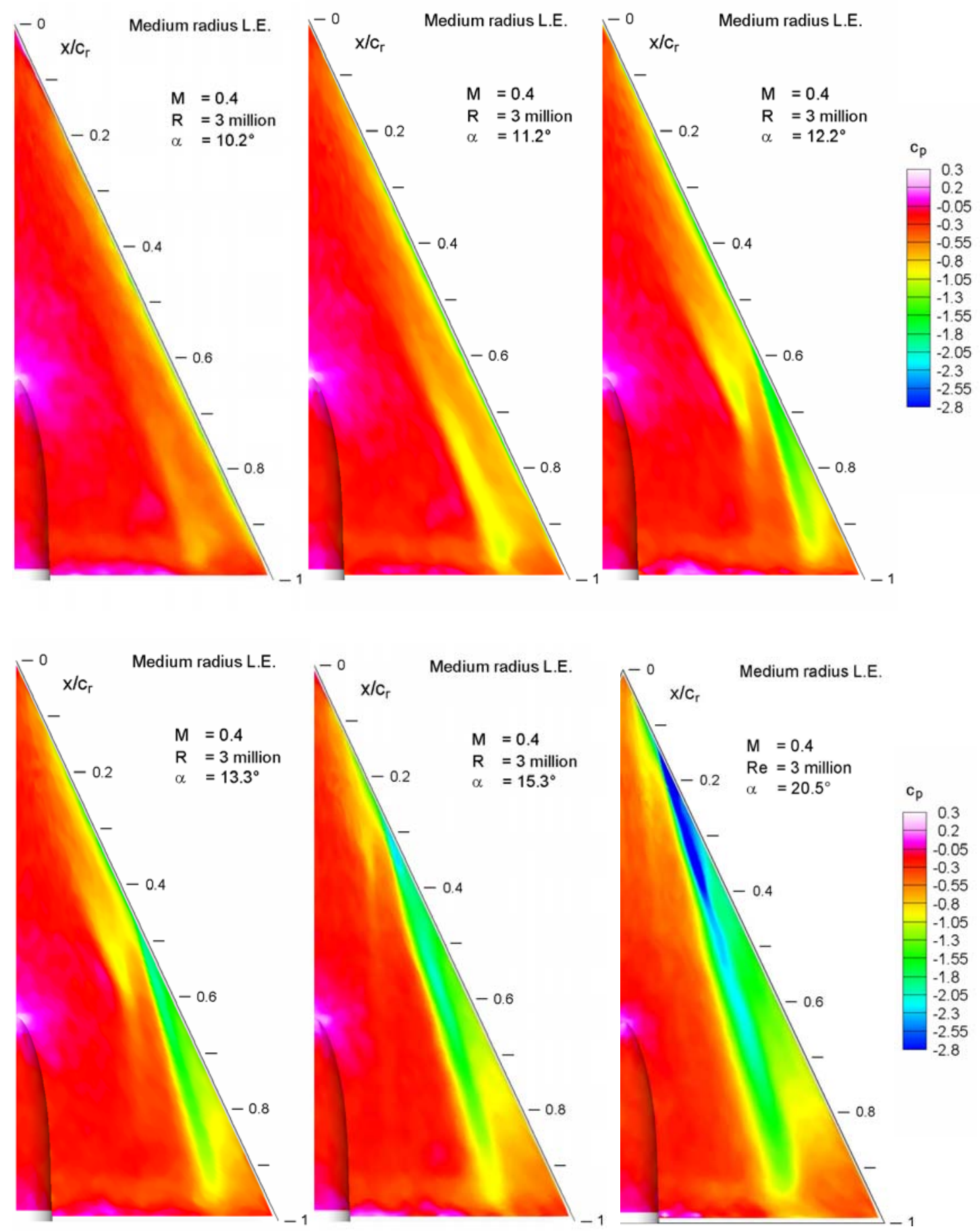

Figure 9. Pressure distributions on the VFE-2 configuration with rounded leading edges (medium radius) for $\mathrm{M}=0.4, \mathrm{R}_{\mathrm{mac}}=3 \cdot 10^{6}$ at various angles of attack from the PSP experiments at DLR Goettingen ${ }^{15,19}$. 


\section{Variation of Reynolds number}

The vortex formation strongly depends on the Reynolds number as shown in Fig. 10 and Fig. 11. With decreasing Reynolds number the onset of the outer primary vortex moves upstream and its strength increases, whereas the inner primary vortex is weakened and its position moves distinctly inboard.
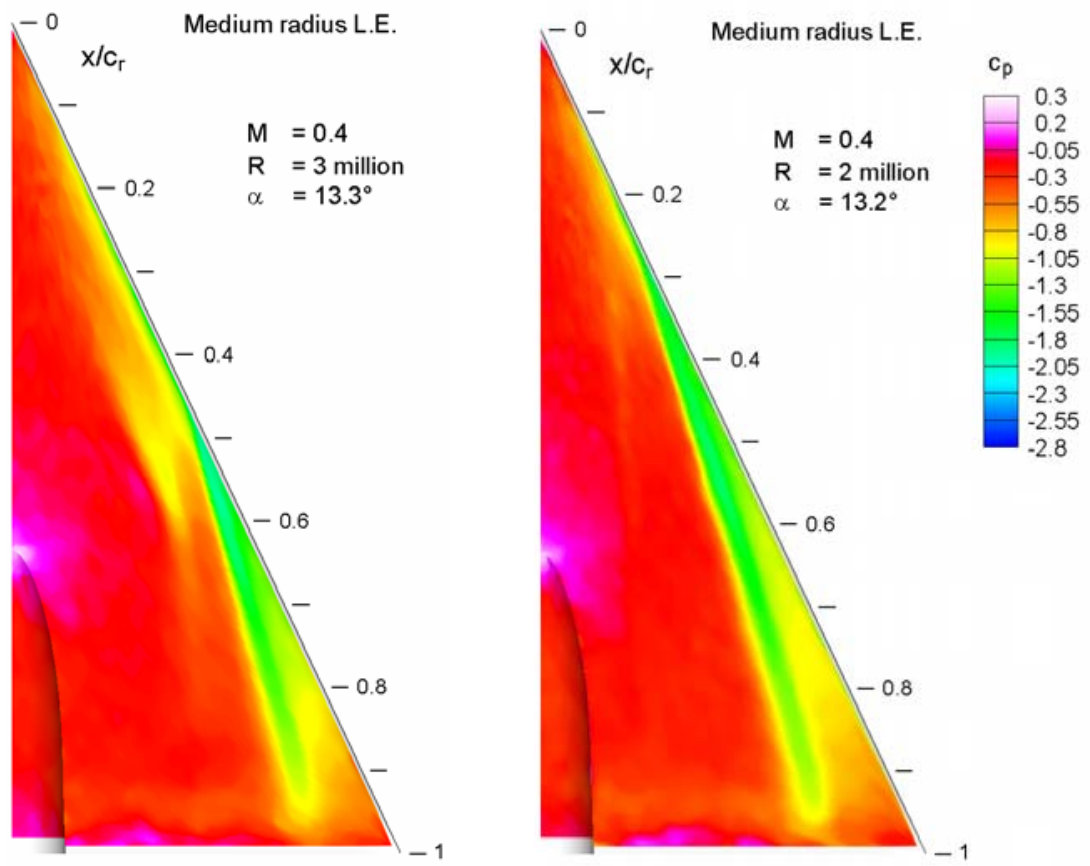

Figure 10. Pressure distributions on the VFE-2 configuration with rounded leading edges (medium radius) for $M=0.4, \alpha=13^{\circ}$ at different Reynolds numbers from the PSP experiments at DLR Goettingen ${ }^{19}$.
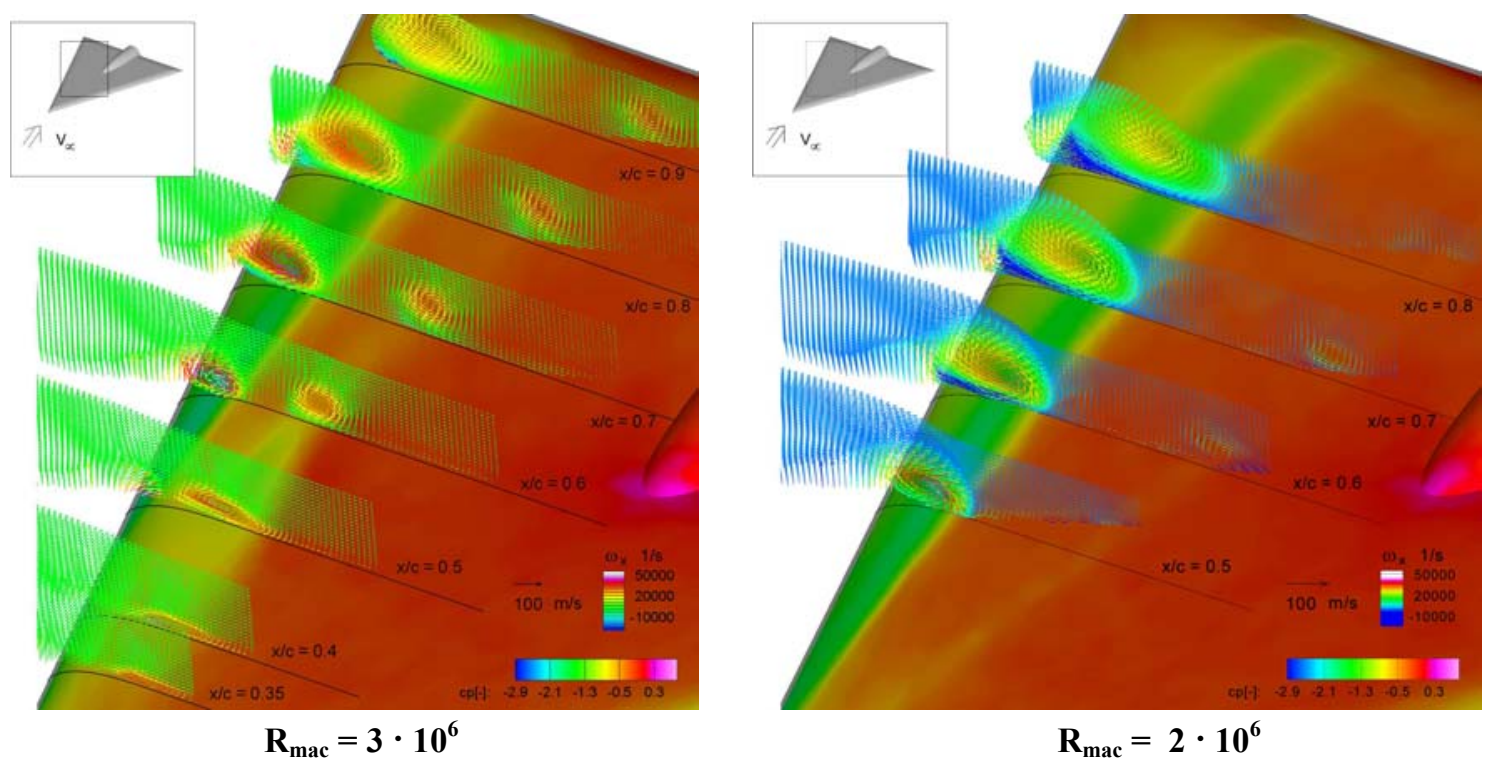

Figure 11. Pressure distributions and flow fields on the VFE-2 configuration with rounded leading edges (medium radius) for $\mathrm{M}=0.4, \alpha=13^{\circ}$ at different Reynolds numbers from the PSP and PIV experiments at DLR Goettingen ${ }^{19}$. 
A reduction in Reynolds number has an analogous effect as an increase of the angle of attack according to Fig. 9, but nevertheless the reasons for this upstream movement of the onset of the outer primary vortex are different for both cases. With increasing angle of attack the adverse pressure gradients on the upper surface of the wing increase as well, and this leads to the upstream movement of the onset of the outer primary vortex. If the angle of attack is unchanged, however, the adverse pressure gradients remain at the same level, but with decreasing Reynolds number the viscous flow is no longer able to stay attached, and this leads again to an upstream movement of the onset of the outer primary vortex.

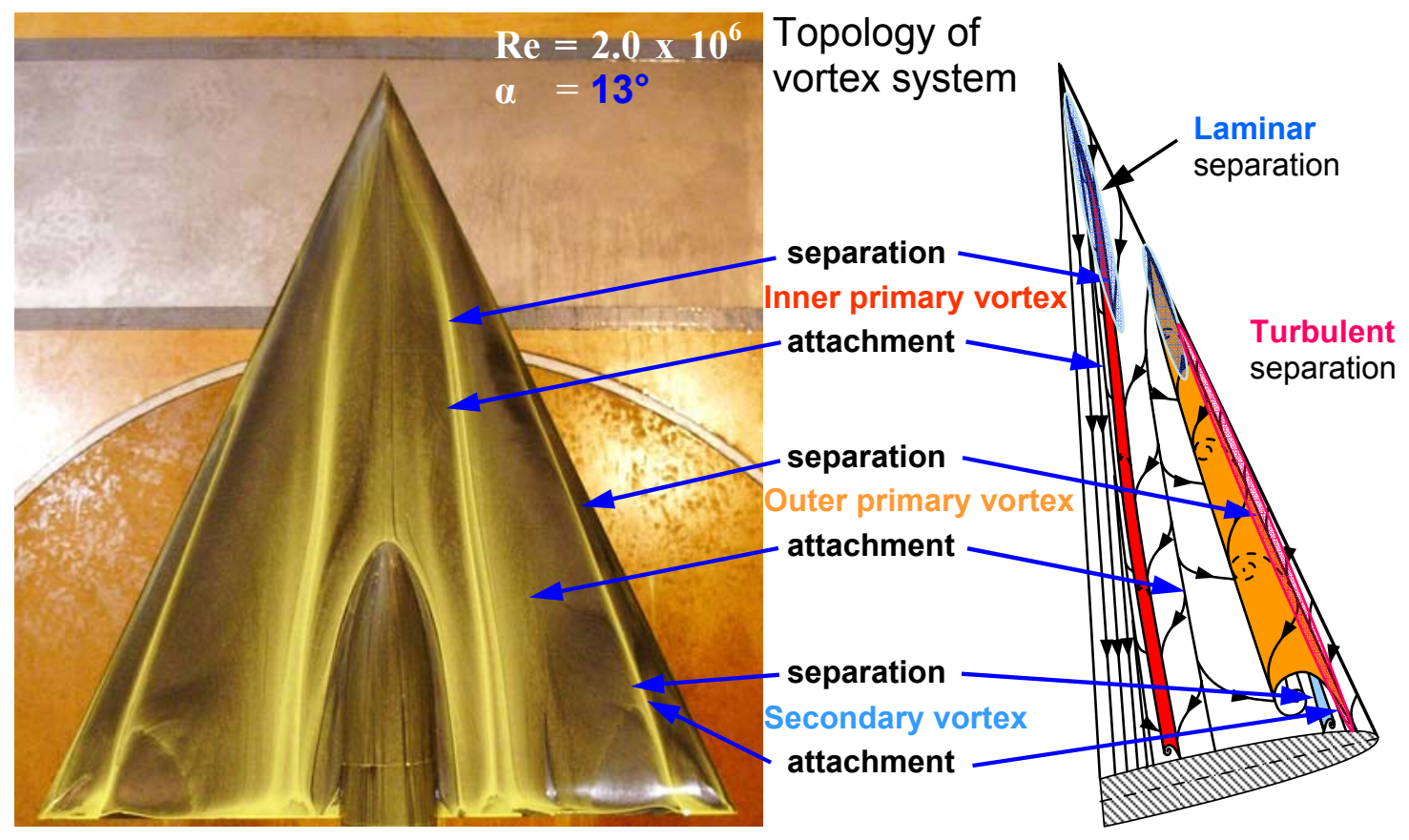

Figure 12. Surface oilflow patterns and flow topology for the VFE-2 configuration with rounded leading edges (medium radius) for $M=0.14, R_{\text {mac }}=2 \cdot 10^{6}, \alpha=13^{\circ}$ from the measurements at TU Munich ${ }^{23,24}$.

The topology of the flow field has also been studied by means of surface oilflow patterns. Fig. 12 shows surface streamlines for $\alpha=13^{\circ}$ and $\mathrm{R}_{\mathrm{mac}}=2 \cdot 10^{6}$. The two primary vortices are clearly indicated and their positions coincide nicely with the result according to Fig. 10. For low Reynolds numbers the inner primary vortex is found in a more inboard position as compared with higher Reynolds numbers.

\section{Variation of Mach number}

In contrasting a subsonic and a transonic Mach number condition, the principal vortex topology remains unchanged, but some compressibility effects can be recognized. In transonic flow the formation of the outer primary vortex starts earlier ${ }^{15}$. Fig. 13 shows the pressure distributions at $\alpha=13^{\circ}$ and $\mathrm{R}_{\mathrm{mac}}=2 \cdot 10^{6}$ for two different Mach numbers. At the higher Mach number the onset of the outer primary vortex has moved slightly upstream and its axis is distinctly shifted inboard. For $\mathrm{M}=0.8$ an inner primary vortex was no longer found. If it exists at all in transonic flow, this vortex must be very weak. The corresponding effect of Mach number on the flow field is further illustrated in Fig. 14. The outer primary vortex is shifted inboard and its flow field is enlarged.

\section{E. Status of the boundary layers at $\alpha=13^{\circ}$}

The 3D vortical flow field has been analyzed by means of hot-wire measurements, in which large portions of the boundary layers were covered. A typical result for incompressible free stream conditions $\left(\mathrm{M}=0.07, \mathrm{R}_{\mathrm{mac}}=1 \cdot 10^{6}\right)$ 

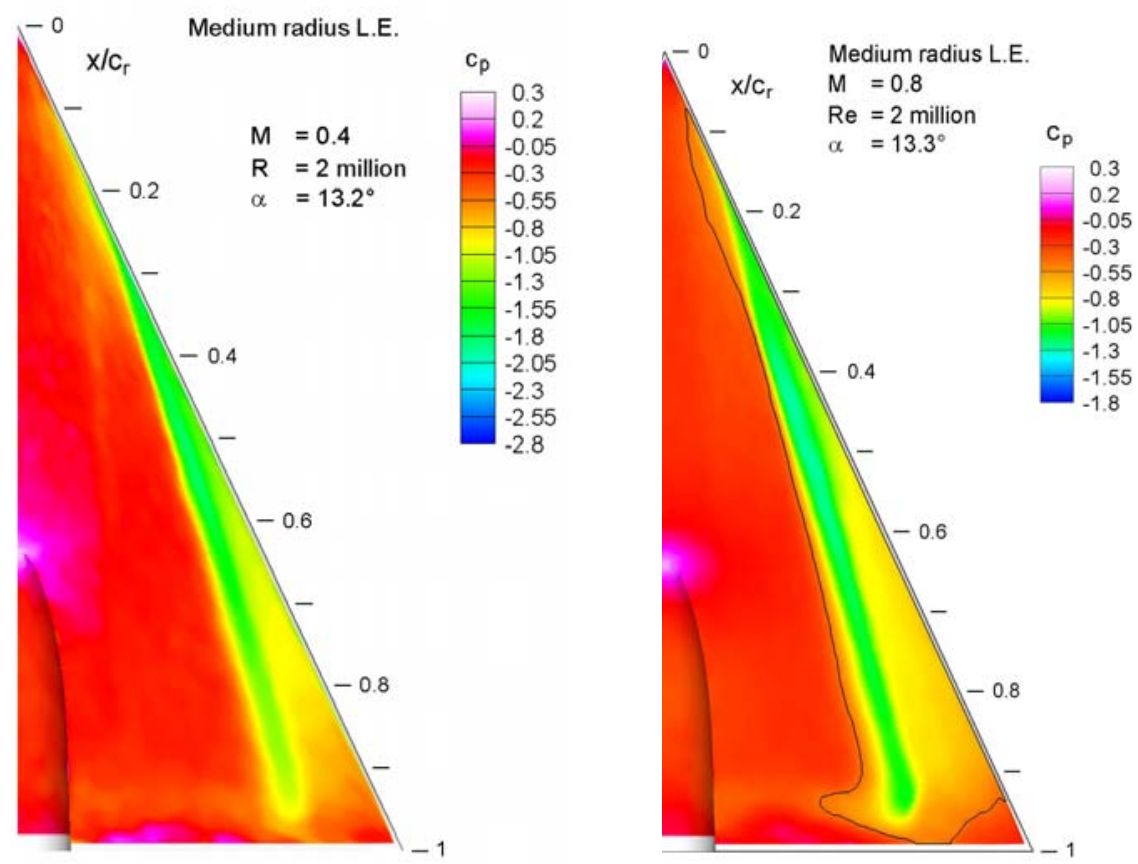

Figure 13. Pressure distributions on the VFE-2 configuration with rounded leading edges (medium radius) at $R_{\text {mac }}=2 \cdot 10^{6}$ and $\alpha=13^{\circ}$ for different Mach numbers from the PSP experiments at DLR Goettingen ${ }^{15}$. The black line indicates the sonic pressure coefficient.

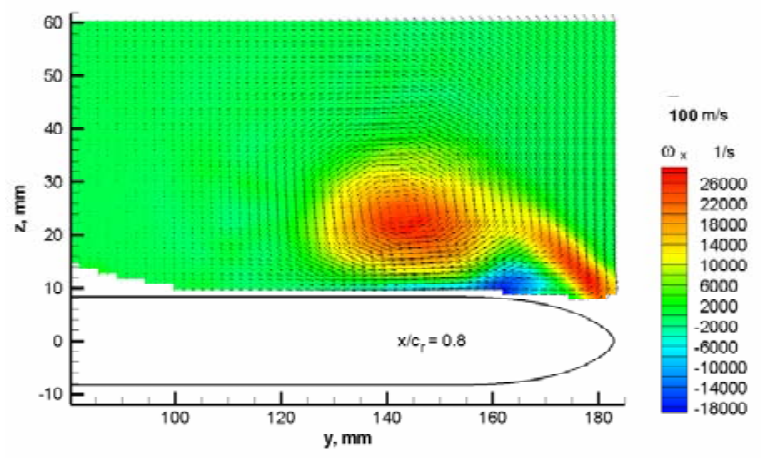

$$
\mathbf{M}=\mathbf{0 . 4}
$$

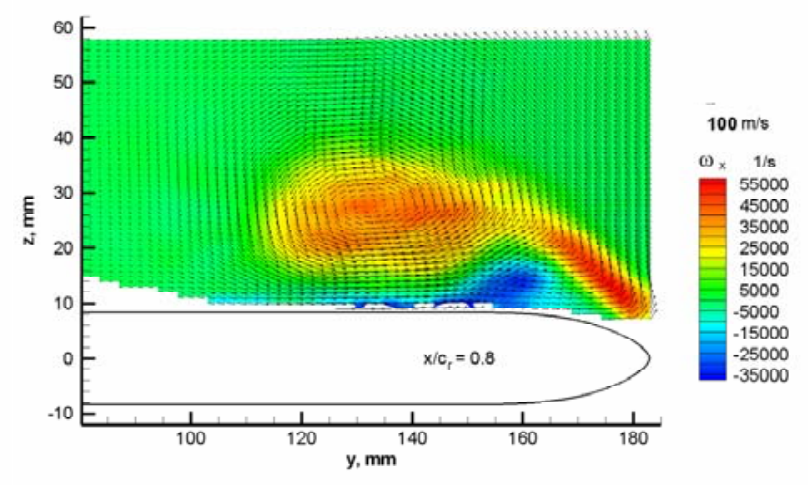

$\mathbf{M}=\mathbf{0 . 8}$

Figure 14. Flow field on the VFE-2 configuration with rounded leading edges (medium radius) at $R_{\text {mac }}=2 \cdot 10^{6}$ and $\alpha=13^{\circ}$ for different Mach numbers from the PIV experiments at DLR Goettingen $^{18}$. Velocity vectors and axial vorticity in the cross $\operatorname{section}$ at $x / c=0.8$.

is shown in Fig. 15. The inner measurement station at $\eta=2 y / b_{\text {loc }}=0.4$ is located inboard of the outer primary vortex attachment line according to Fig. 12 and it is thus related to the inner primary vortex. At this station the boundary layer is very thick $\left(\zeta=2 z / b_{\text {loc }}=0.025\right)$ as indicated by the time-averaged longitudinal velocity component $\bar{u} / U_{\infty}$. Outside of the boundary layer the fluctuations of almost all velocity components increase towards the wing surface with the only exception of a slight decrease in $\sqrt{\overline{\left(w^{\prime}\right)^{2}}} / U_{\infty}$, and within the boundary layer all fluctuations increase rapidly towards the wall. 

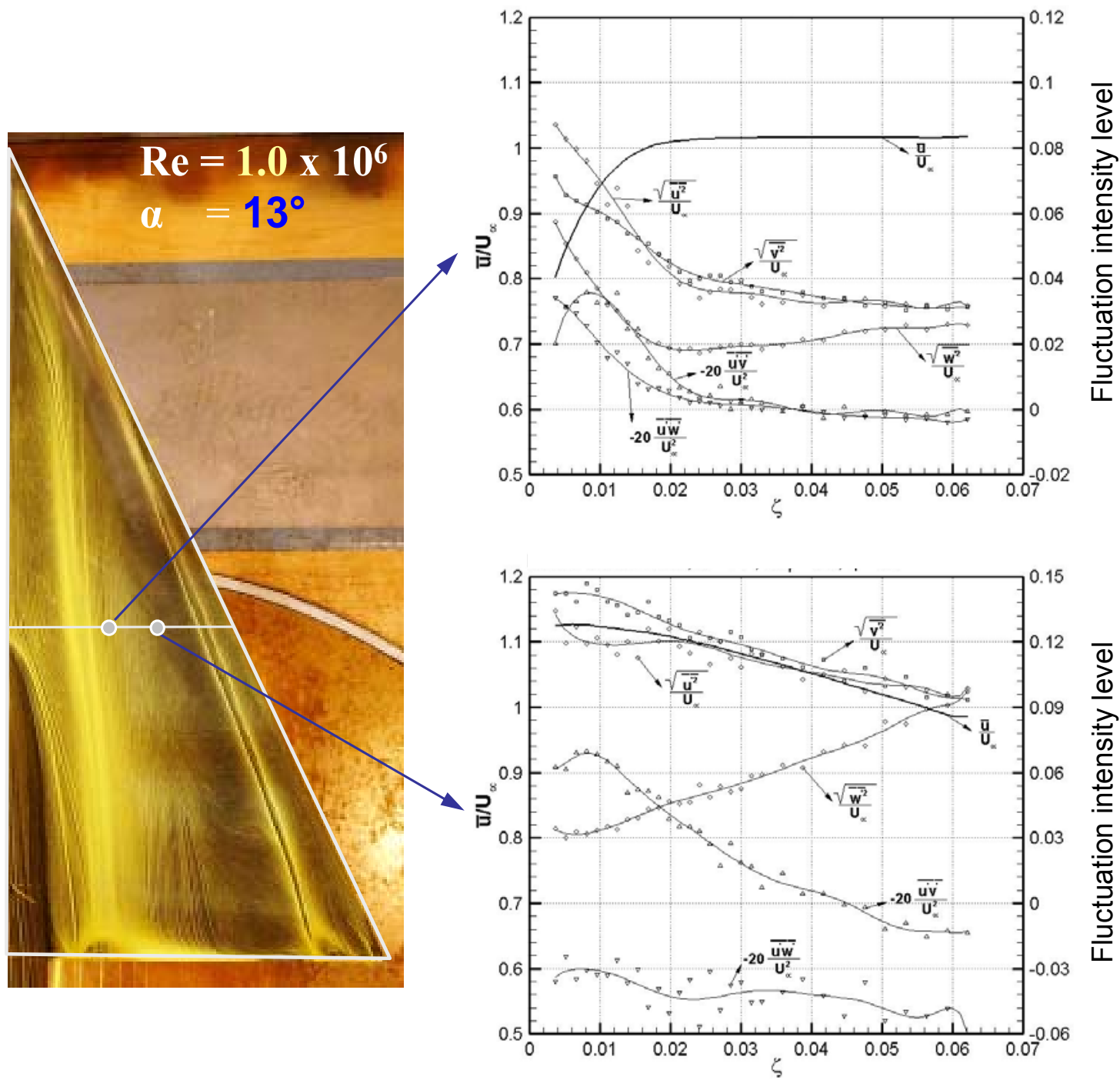

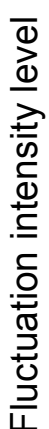

Figure 15. Results of boundary layer measurements on the VFE-2 configuration with rounded leading edges (medium radius) for $M=0.07, R_{\text {mac }}=1 \cdot 10^{6}$ and $\alpha=13^{\circ}$ at two stations (top $\eta=0.4$, bottom $\eta=0.6$ ) in the section $x / c_{r}=0.6$ from the hot-wire anemometry at TU Munich ${ }^{11}$.

The same applies for the outer measurement station at $\eta=2 \mathrm{y} / \mathrm{b}_{\mathrm{loc}}=0.6$, but the boundary layer is much thinner there $\left(\zeta=2 \mathrm{z} / \mathrm{b}_{\text {loc }}=0.008\right)$. The outer measurement station is located outboard of the outer primary vortex attachment line. Thus an accelerated outboard flow underneath the outer primary vortex is present, which leads to the strong decrease of the boundary layer thickness. Simultaneously measured pressure fluctuations on the wing surface according to Fig. 16 indicate, that the flow in the region of the outer primary vortex is distinctly turbulent. At the inner measurement station, however, the boundary layer status remains unclear: At the wall almost no pressure fluctuations were found (Fig. 16), whereas in the boundary layer some fluctuations are present. 


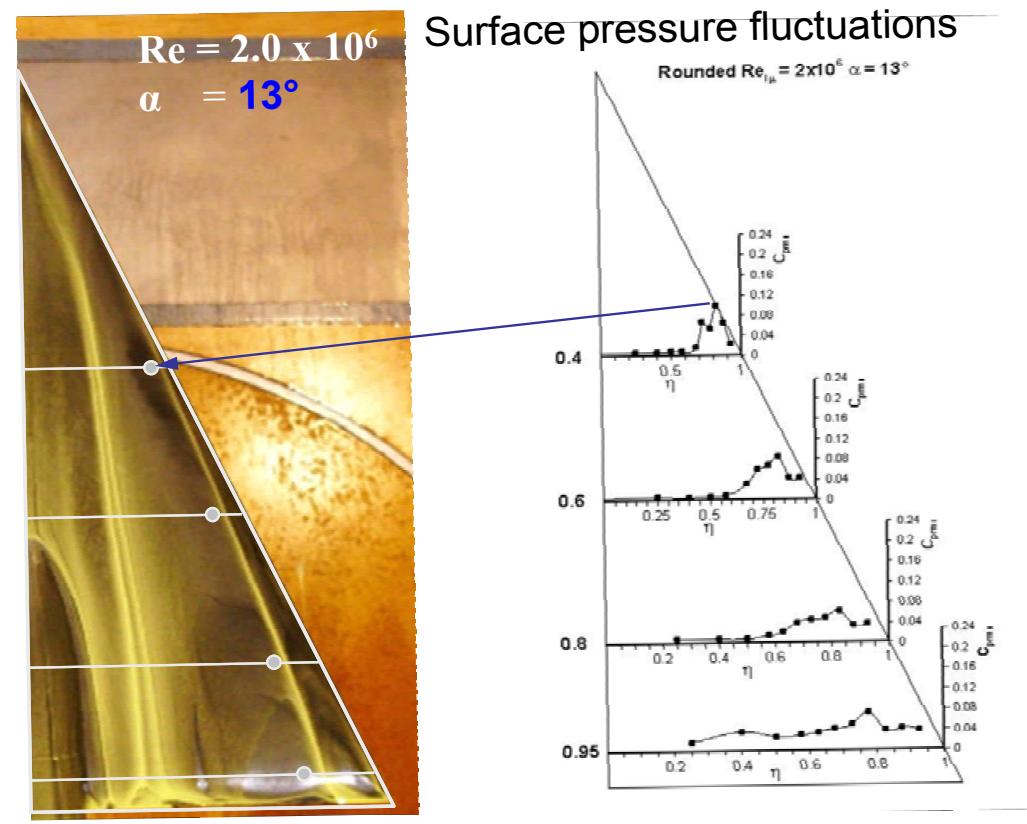

Figure 16. Pressure fluctuations on the VFE-2 configuration with rounded leading edges (medium radius) for $M=0.14, R_{\mathrm{mac}}=2 \cdot 10^{6}$ and $\alpha=13^{\circ}$ in four sections from the measurements at TU Munich ${ }^{11}$.

\section{Fully developed vortical flow without vortex breakdown}

For the sharp edged configuration a single primary vortex occurs along the whole leading edge from moderate to high angles of attack. For the blunt edged configuration (medium radius) an inner and an outer primary vortex are formed in the same angle of attack range as described in section III. With increasing angle of attack the outer primary vortex becomes more and more dominant along the whole leading edge, whereas the inner primary vortex is weakened.

At $\alpha=18^{\circ}$ in both cases a fully developed vortical flow with a single primary vortex is present according to Fig. 17. For the rounded leading edge configuration only very weak remnants of an inner primary vortex can be detected for the low Mach number case. The pressure distributions for both Mach numbers indicate that the primary vortex on the configuration with blunt leading edge is located slightly more outboard than for the configuration with sharp leading edge, and some differences concerning the suction magnitude can also be recognized, but the overall behavior of the flow is very similar. Although difficult to see, this is also demonstrated in Fig. 18 for the two flow fields through PIV results for the time-averaged velocities in various cross sections. 


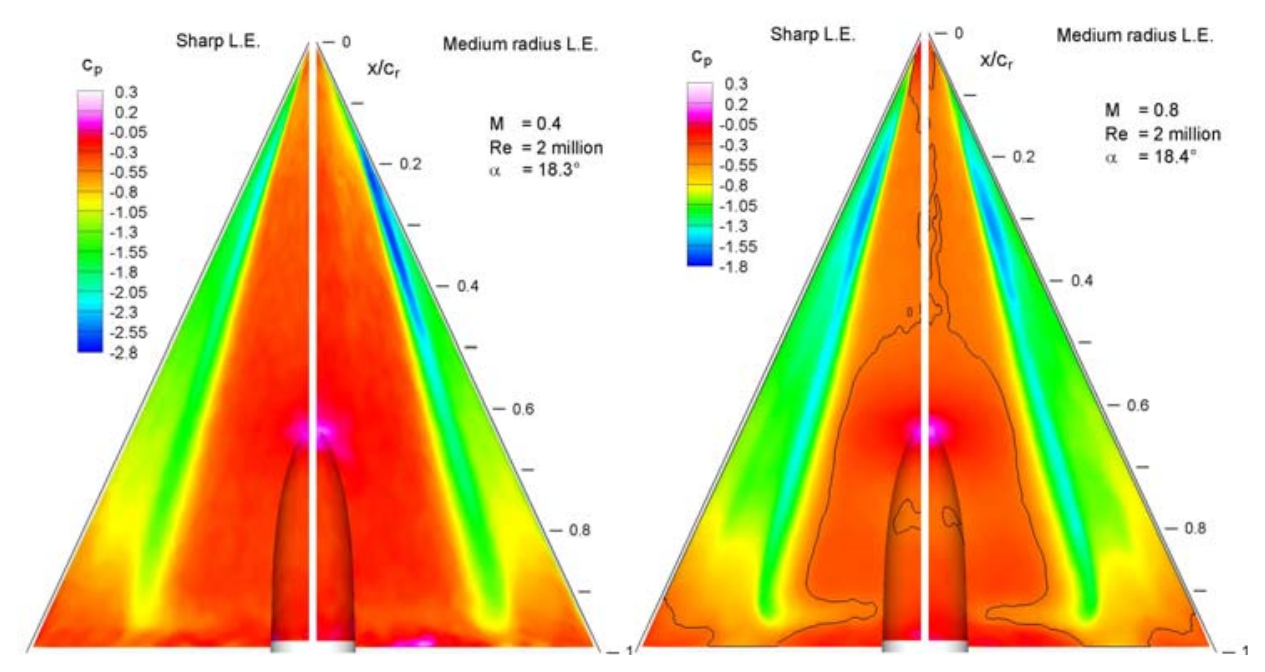

Figure 17. Pressure distributions on the VFE-2 configuration with sharp and rounded leading edges (medium radius) at $R_{\text {mac }}=2 \cdot 10^{6}$ and $\alpha=18^{\circ}$ for two Mach numbers according to the PSP experiments at DLR Goettingen ${ }^{15}$. The black line indicates the sonic pressure coefficient.

\section{Sharp Leading Edge}

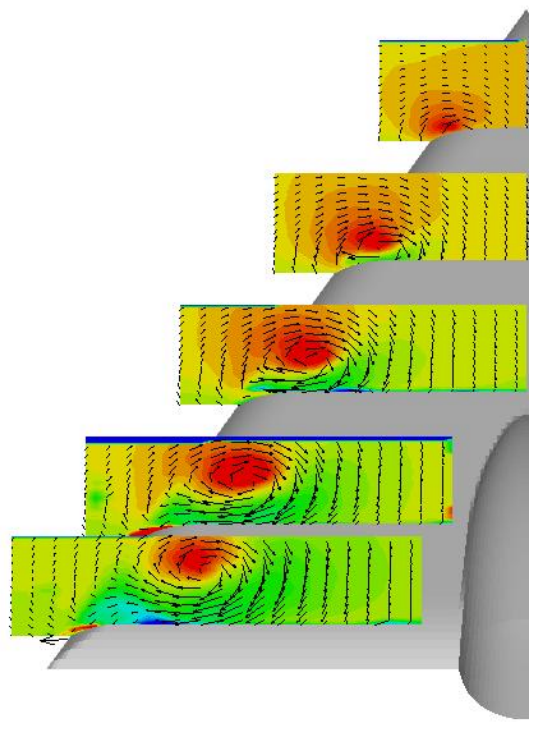

\section{Rounded Leading Edge}

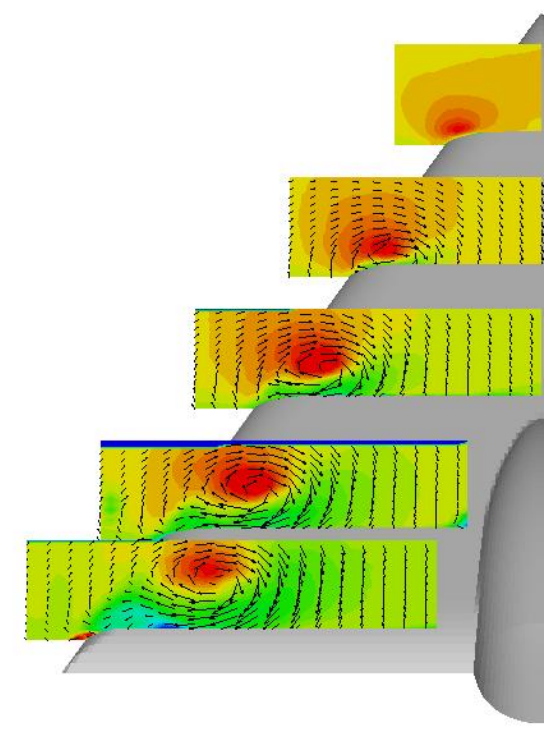

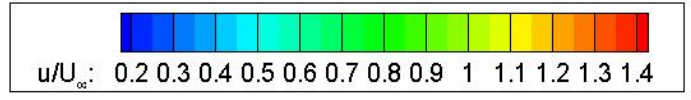

Figure 18. Flow field around the VFE-2 configuration with sharp and rounded leading edges (medium radius) at $M \approx 0.1, R_{\text {mac }}=1 \cdot 10^{6}, \alpha=18^{\circ}$. PIV results from TU Munich ${ }^{25}$ for the timeaveraged velocity in various cross sections: $x / c_{r}=0.2,0.4,0.6,0.8,0.95$. 
Within VFE-2 the case $\alpha=18^{\circ}$ with a well developed single primary vortex will be used to check the various turbulence models for their proper description of vortical flow. For this purpose hot-wire anemometry (HWA) has also been applied at TU Munich. Concerning the time averaged velocity components $\overline{\mathrm{u}}, \overline{\mathrm{v}}, \overline{\mathrm{w}}$ the results according to Fig. 18 have been confirmed with these measurements. In addition, however, the complete field of the velocity fluctuations u', v', w' is now available. As examples Fig. 19 shows the measured fluctuations of the velocity component $\mathrm{u}_{\mathrm{rms}} / \mathrm{U}_{\infty}$ as well as $\overline{\mathrm{u}^{\prime} \mathrm{w}^{\prime}} / \mathrm{U}_{\infty}{ }^{2}$ in the cross section plane at $\mathrm{x} / \mathrm{c}=0.6$. Again there are only minor differences between the measurements for sharp and for medium radius rounded leading edges, but from the full field of the fluctuations $u^{\prime}, v^{\prime}, w^{\prime}$ the distributions of the turbulent kinetic energy and the eddy viscosity can be determined. Corresponding evaluations of the experiments are presently under way, and the results will be used for comparisons with numerical simulations of the vortical flow field by means of various turbulence models.

$$
\text { Sharp Leading Edge }
$$
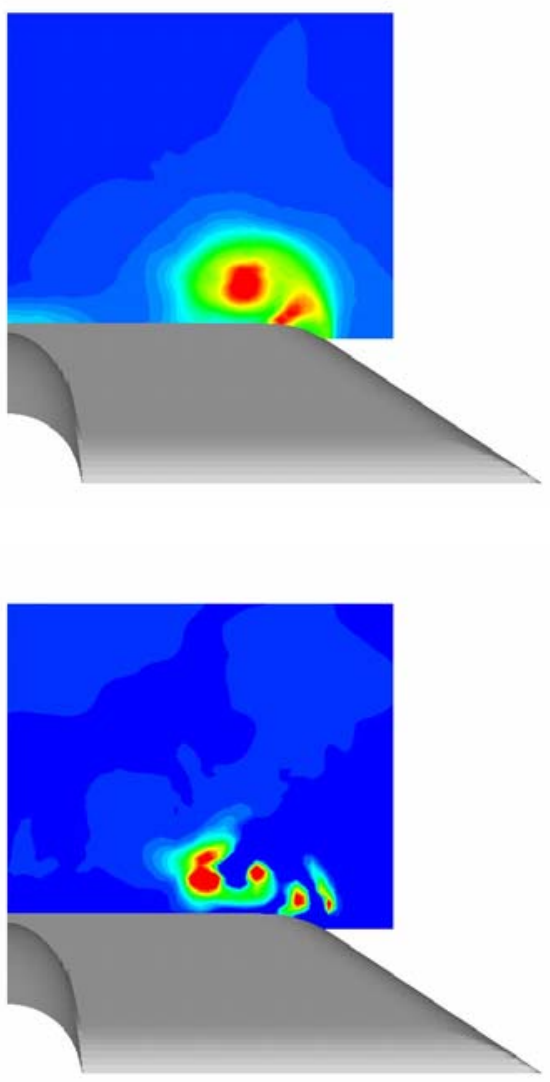

Rounded Leading Edge
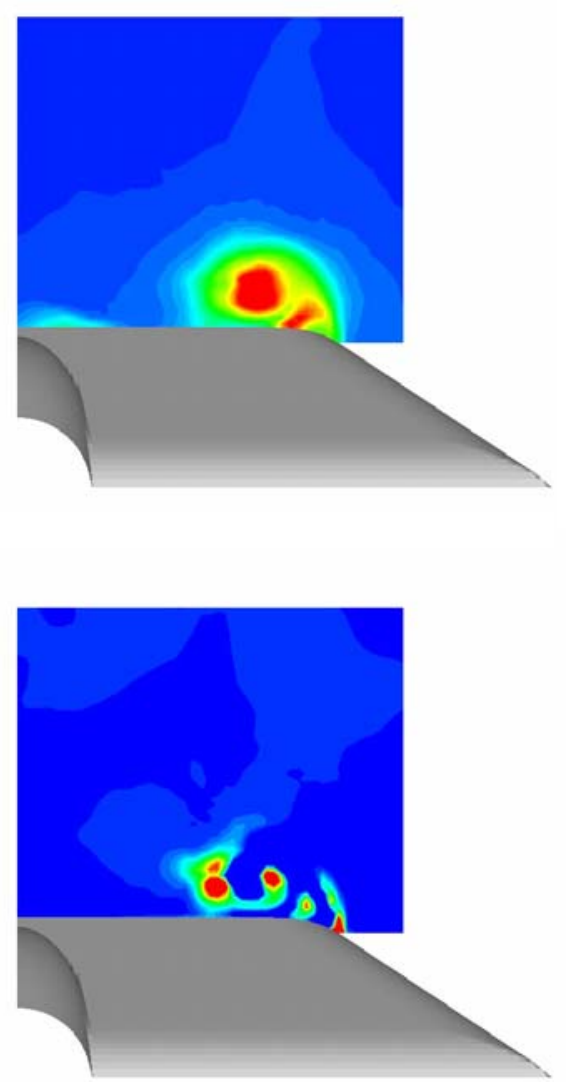
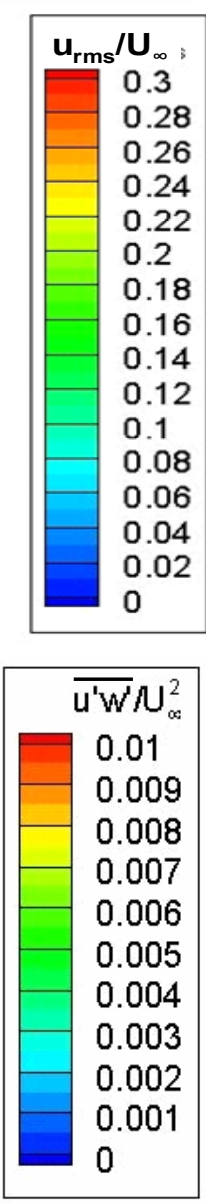

Figure 19. Flow field around the VFE-2 configuration with sharp and rounded leading edges (medium radius) at $\mathrm{M} \approx 0.1, \mathrm{R}_{\mathrm{mac}}=1 \cdot 10^{6}, \alpha=18^{\circ}$. HWA results from TU Munich ${ }^{25}$ for the velocity fluctuations $u_{\mathrm{rms}} / \mathrm{U}_{\infty}$ (top) and $\overline{u^{\prime} w^{\prime}} / \mathbf{U}_{\infty}{ }^{2}($ bottom) in the plane at $\mathbf{x} / \mathbf{c}=0.6$.

The unsteadiness of the flow can also be evaluated from these experiments by determining the power spectrum density distribution of any fluctuating flow quantity as function of the frequency at any measured position of the flow field. An example of this kind is shown in Fig. 20 for the sharp leading edge configuration. In the left-hand graph the distribution of the fluctuations of the u-component of the velocity $\mathrm{u}_{\mathrm{rms}} / \mathrm{U}_{\infty}$ shown in Fig. 19 (upper left) is given. On the right side of Fig. 20 the power spectrum density distribution for the velocity component $u / U_{\infty}$ is shown at a location near the vortex core (indicated by the large dot on the left side of Fig. 20). In this turbulent flow near the vortex centre a large variety of frequencies is present with distinctly high values at low frequencies. 

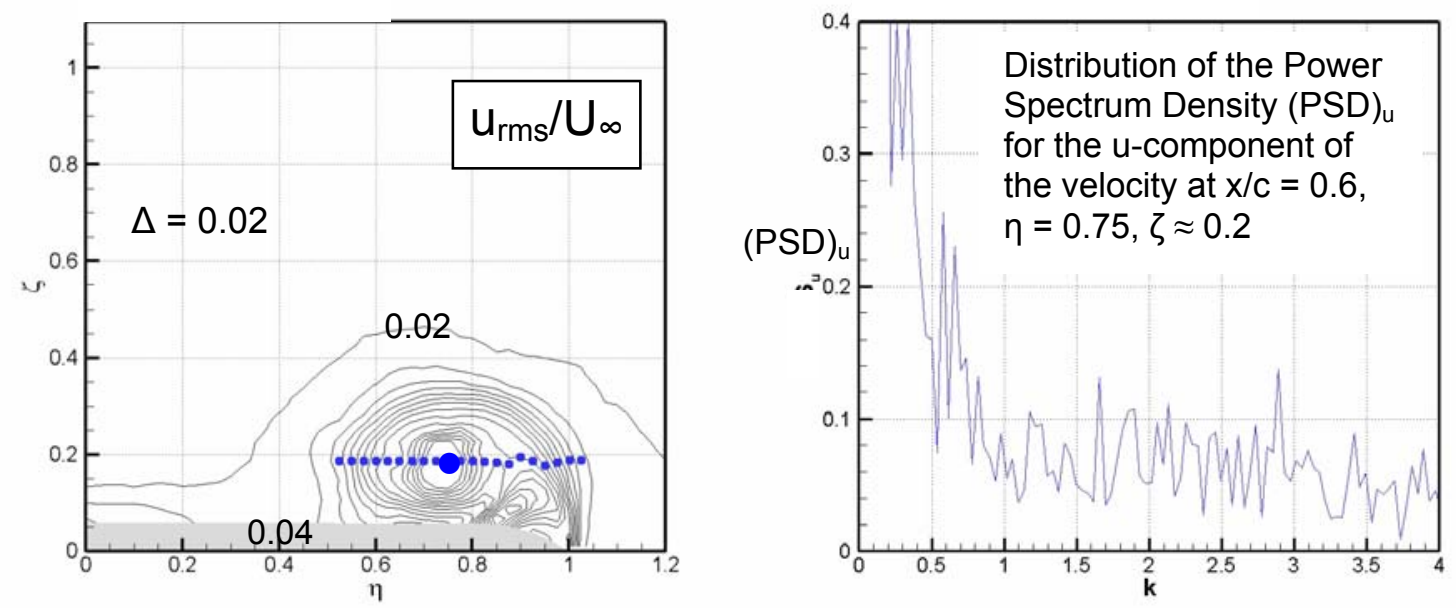

Figure 20. Flow field around the VFE-2 configuration $\left(65^{\circ}\right.$ delta wing) with sharp leading edges at $M \approx 0.1, R_{\text {mac }}=1 \cdot 10^{6}, \alpha=18^{\circ}$. Power spectrum density (PSD) $)_{u}$ for the $u$-component of the velocity as function of the reduced frequency $\mathrm{k}$ at $\mathrm{x} / \mathrm{c}=0.6, \eta=0.75, \zeta \approx 0.2$ according to HWA at TU Munich ${ }^{25}$. $\Delta$ denotes contour interval

\section{Fully developed vortical flow with vortex breakdown}

At large angles of attack vortex breakdown takes place in the primary vortices. Today it is common understanding that the flow past slender delta wings at large angles of attack becomes unsteady even for a fixed wing. This means that for large angles of attack and steady boundary conditions only unsteady solutions of the governing equations do exist. The spiral-type vortex breakdown is well predicted by numerical solutions of the Navier-Stokes equations ${ }^{26,27}$ : The instantaneous vortex axis spirals in space against the sense of rotation of the primary vortex and this spiral rotates in the sense of the primary vortex, and in the center of the spiraling motion a region of reversed flow is present. Correspondingly all quantities of the flow field show oscillations and on the surface of the wing an unsteady pressure distribution is present.

The unsteadiness due to vortex breakdown starts in a region with steep pressure gradients in streamwise direction, and this region is usually located in the vicinity of the trailing edge of the wing. As angle of attack is increased the extent of unsteady vortical flow increases in size and the vortex breakdown onset point moves upstream. In the rear part of the wing, which is directly influenced by the unsteadiness of the flow in the primary vortices, the time-averaged pressure distributions generally show distinct reductions of suction, and this leads to a certain loss of lift.

For the VFE-2 configuration vortex breakdown in the primary vortex has already progressed over the wing at $\alpha=23^{\circ}$ for sharp and for medium radius rounded leading edges. For both configurations the unsteady vortical flow field has been measured at TU Munich by means of unsteady surface pressure measurements and by PIV and HWA investigations. For the configuration with medium radius rounded leading edges Fig. 21 shows the surface pressure fluctuations $\left(\mathrm{c}_{\mathrm{p}}\right)_{\mathrm{rms}}$ at four cross sections of the wing, and for a certain station in each of these distributions the amplitude spectrum density $(\mathrm{SD})_{\mathrm{cp}}$ of the pressure fluctuations is given. The high values of the fluctuations around the reduced frequency $\mathrm{k} \approx 1.3$ in the last section are caused by the spiral motion of vortex breakdown.

In these cross sections the time-averaged flow field has been measured at TU Munich by means of PIV investigations and the complete unsteady flow field has been determined using the HWA technique. For the configuration with medium radius rounded leading edge Fig. 22 shows the unsteadiness of the flow field with vortex breakdown in the cross section at $\mathrm{x} / \mathrm{c}=0.8$. The annular form of the area with high fluctuations is typical for the spiral mode of vortex breakdown. Further analysis according to Figs. 21 and 22 will lead to experimental data for the frequency of the spiral mode of vortex breakdown. Evaluations of this kind are presently under way and comparisons with numerical results are prepared. 

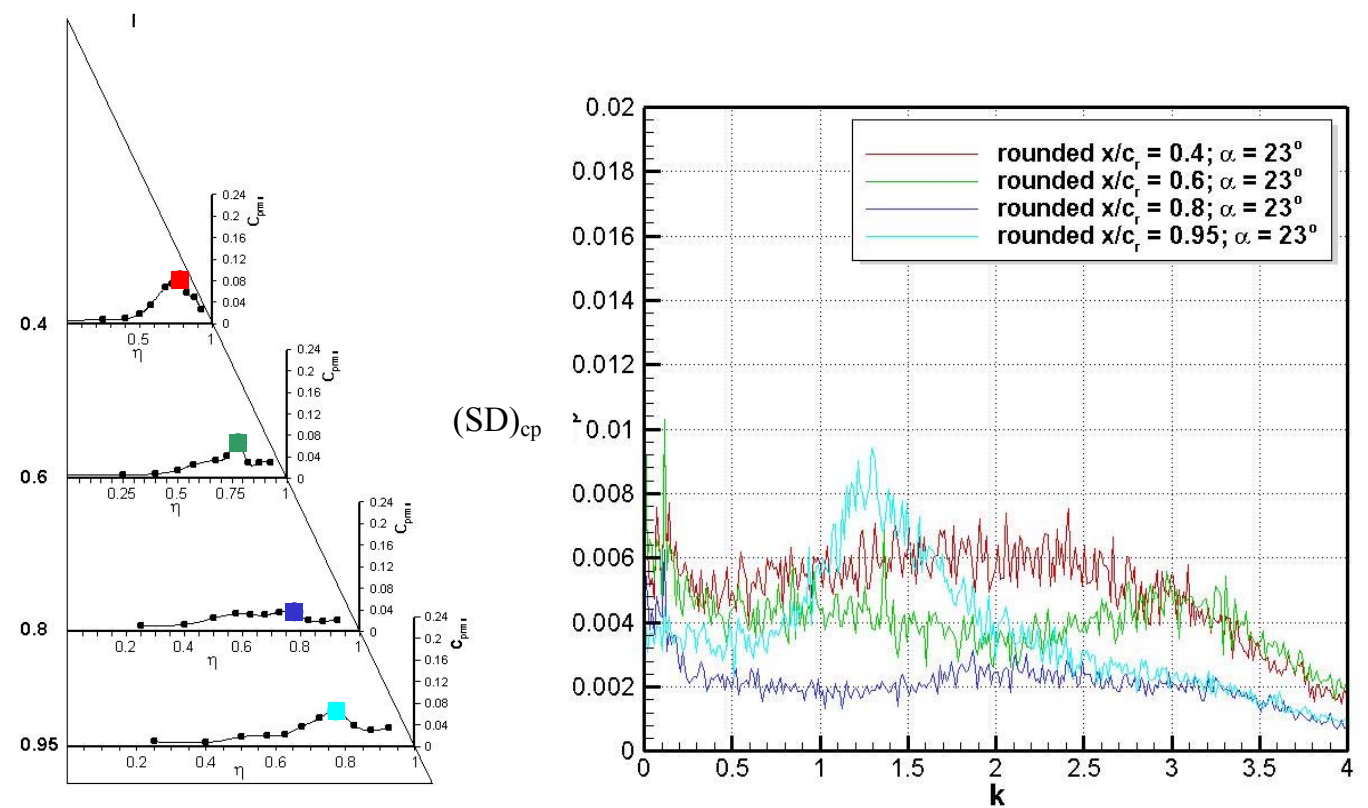

Figure 21. Flow field around the VFE-2 configuration with medium radius rounded leading edges at $M=0.14, R_{\text {mac }}=2 \cdot 10^{6}, \alpha=23^{\circ}$. Surface pressure fluctuations $\left(c_{\mathrm{p}}\right)_{\mathrm{rms}}$ and spectrum density $(\mathrm{SD})_{\mathrm{cp}}$ for certain stations in four cross sections according to measurements at TU Munich ${ }^{25}$
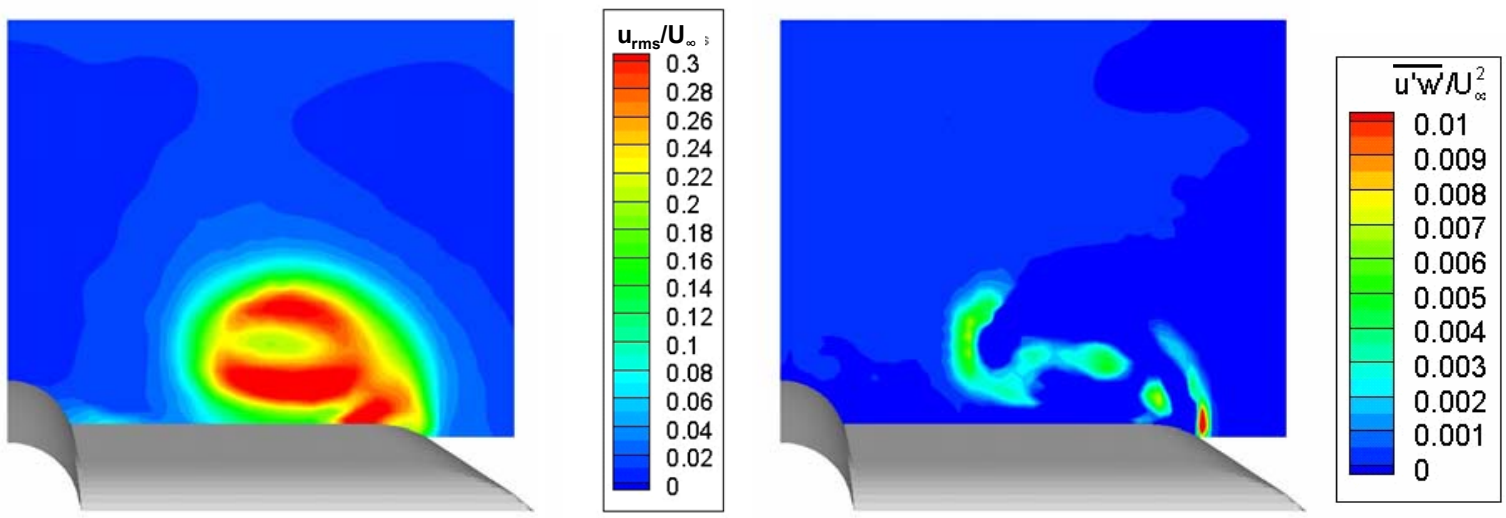

Figure 22. Flow field around the VFE-2 configuration with medium radius rounded leading edges at $M \approx 0.1$, $R_{\text {mac }}=1 \cdot 10^{6}, \alpha=23^{\circ}$. HWA results from TU Munich ${ }^{25}$ for the velocity fluctuations $u_{r m s} / U_{\infty}$ and $\overline{u^{\prime} w^{\prime}} / U_{\infty}{ }^{2}$ in the cross section at $x / c=0.8$

Fig. 23 shows PSP measured pressure distributions for sharp and (medium radius) rounded leading edges at $\alpha=$ $25^{\circ}$ and $\mathrm{R}_{\mathrm{mac}}=2 \cdot 10^{6}$. For both Mach numbers there exist moderate differences between the results for sharp and blunt leading edges. At the lower Mach number $\mathrm{M}=0.4$ (left-hand part) no supersonic zones and no shock waves exist for an angle of attack of $\alpha=25^{\circ}$. At this angle of attack vortex breakdown would certainly be ahead of $\mathrm{x} / \mathrm{c}=$ 0.8 , given the results just discussed with Fig. 21 and Fig 22, although the precise breakdown location is in general difficult to discern from subcritical surface pressure information.

At constant angle of attack and with the increase to a transonic Mach number, however, a distinct change of the pressure distribution on the wing can be taken from the results for $\mathrm{M}=0.8$ (right-hand part). At this transonic Mach number a local supersonic zone with a terminating shock wave is formed in the vicinity of the sting mount. The adverse pressure jump in the shock wave causes additional unsteadiness in the vortical flow field, and most likely shifts vortex breakdown upstream. 


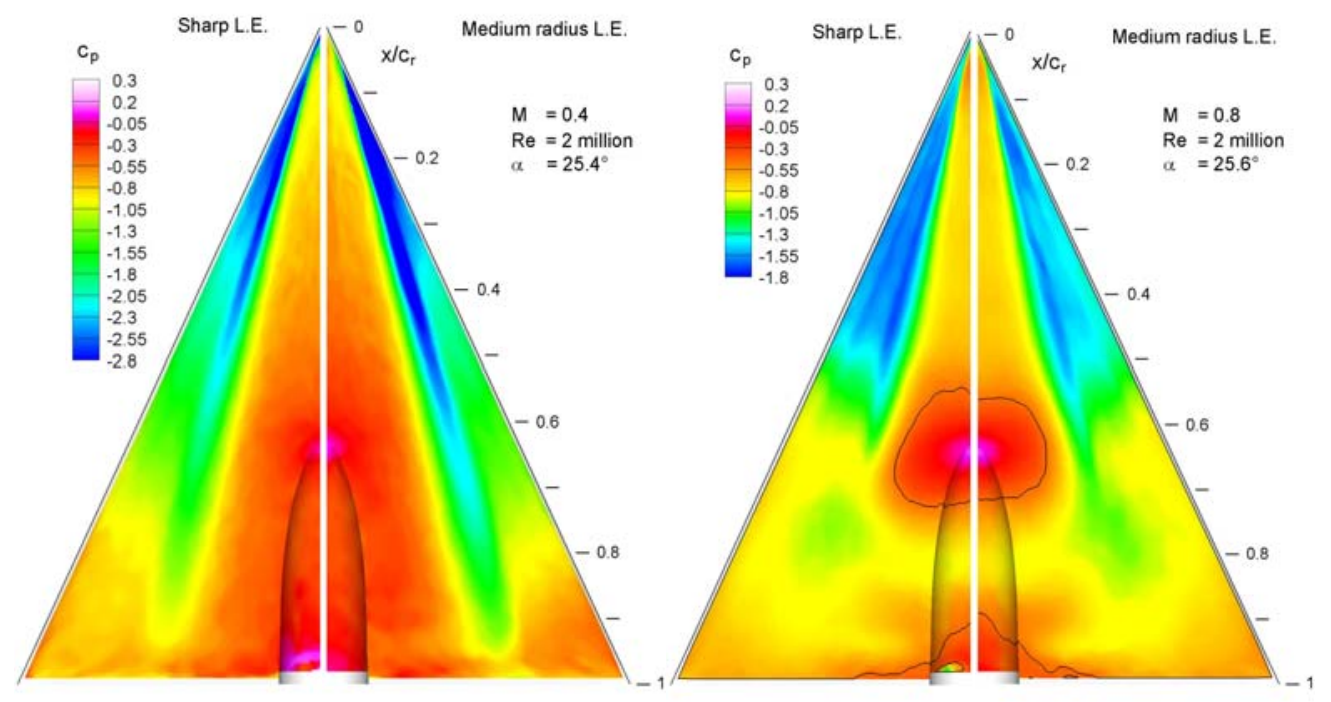

Figure 23. Pressure distributions on the VFE-2 configuration with sharp and rounded leading edges (medium radius) at $R_{\mathrm{mac}}=2 \cdot 10^{6}$ and $\alpha=25^{\circ}$ for different Mach numbers according to the PSP experiments at DLR Goettingen ${ }^{15}$. The black line indicates the sonic pressure coefficient.

\section{Concluding Remarks}

At the onset of Vortex Flow Experiment 2 an initial data base had been established by NASA to examine the effects of Mach number, Reynolds number, angle of attack, and leading-edge bluntness on separation-induced leading-edge vortex flows common to slender wings. The data spanned a wide range of Mach and Reynolds numbers in such a way to isolate Mach and Reynolds number effects for four different leading-edge bluntness values. Measurements were limited to fairly detailed static surface pressure coefficients as well as to the normal force and pitching moment coefficients. These experiments demonstrated significant effects of the parameters studied and also indicated a multiple-vortex characteristic of the leading-edge vortex separation from the blunt leading edge different from that observed with sharp leading edges.

As a result of VFE-2 significant details of this flow have now been measured. This was accomplished through 15 new wind tunnel experiments coordinated among four countries. A focus was established on two of the leading edges (sharp and medium bluntness) from the NASA experiments, and four new wind tunnel models were fabricated to support the new testing. Surface and off-body measurements, for both steady and fluctuating quantities, have been accumulated using a wide variety of test techniques as well as six-component forces and moments.

The experimental campaigns confirm the multiple-vortex structure of blunt leading edge vortex separation and considerably more detail of this flow is now available through the use of pressure sensitive paints. Detailed flow field measurements provide new insight into this vortical structure, and surface and off-body fluctuating measurements show the turbulent structure of these flows. Surface transition measurements have been very difficult to accomplish, and to date the evaluation of these measurements is not finished. The state of transition still needs to be documented for these flows. The data from VFE 2 present a significant opportunity for further experimental analysis as well as comparison with computational fluid dynamics.

\section{Acknowledgments}

The authors wish to express their appreciation to the following research establishments for having built (or put on loan) new wind tunnel models and for having provided wind tunnels, experimental techniques and staff to carry out theses experimental investigations: NASA Langley Research Center (USA), DLR and TU Munich (DEU), ONERA (FRA), University of Glasgow (GBR) and TUBITAK-SAGE (TUR). Finally, the authors also wish to express their appreciation to the Research and Technology Organization's Applied Vehicle Technology panel (RTO/AVT) for sponsoring these Vortex Flow Experiment 2 activities. 


\section{References}

${ }^{1}$ Chu, J., and Luckring, J. M.,"Experimental Surface Pressure Data Obtained on $65^{\circ}$ Delta Wing Across Reynolds Number and Mach Number Ranges," NASA TM 4645, 1996.

${ }_{2}^{2}$ Luckring, J. M., "Reynolds Number and Leading-Edge Bluntness Effects on a $65^{\circ}$ Delta Wing," AIAA Paper 2002-0419, 2002.

${ }^{3}$ Luckring, J. M., "Transonic Reynolds Number and Leading-Edge Bluntness Effects on a $65^{\circ}$ Delta Wing," AIAA Paper 2003-0753, 2003.

${ }^{4}$ Luckring, J. M., "Compressibility and Leading-Edge Bluntness Effects for a $65^{\circ}$ Delta Wing," AIAA Paper 2004-0765, 2004.

${ }^{5}$ Luckring, J. M., "Reynolds Number, Compressibility, and Leading-Edge Bluntness Effects on Delta-Wing Aerodynamics," ICAS Proceedings Yokohama 2004, Paper 4.1.4, 2004.

${ }^{6}$ Hummel, D., and Redeker, G., "A New Vortex Flow Experiment for Computer Code Validation," RTO AVT Symposium Vortex Flow and High Angle of Attack Aerodynamics, Loen, Norway, 7-11 May 2001, Meeting Proceedings RTO-MP-069 (I), SYA 8-1 to 8-31, 2003.

${ }^{7}$ Hummel, D., "Review of the Second International Vortex Flow Experiment (VFE-2)," AIAA Paper 2008-0377, 2007.

${ }^{8}$ Luckring, J. M., "Initial Experiments and Analysis of Vortex Flow on Blunt Edged Delta Wings," AIAA Paper 2008-0378, 2007.

${ }^{9}$ Konrath, R., Klein, Ch. and Schroeder, A., "PSP and PIV Investigations on the VFE-2 Configuration in Sub- and Transonic Flow," AIAA Paper 2008-0379, 2007.

${ }^{10}$ LeRoy, J. F., Rodriguez, O. and Kurun, S., "Experimental and CFD Contribution to Delta Wing Vortical Flow Understanding," AIAA Paper 2008-0380, 2007.

${ }^{11}$ Furman, A. and Breitsamter, Ch., "Turbulent and Unsteady Flow Characteristics of Delta Wing Vortex Systems," AIAA Paper 2008-0381, 2007.

${ }^{12}$ Coton, F., Mat, S. and Galbraith, R., "Low Speed Wind Tunnel Characterization of the VFE-2 Wing," AIAA Paper 20080382, 2007.

${ }^{13}$ Hummel, D., "Effects of Boundary Layer Formation on the Vortical Flow Above Slender Delta Wings," RTO AVT Symposium Enhancement of NATO Military Flight Vehicle Performance by Management of Interacting Boundary Layer Transition and Separation, Prague, Czech Republic, 4-8 Oct 2004, Meeting Proceedings RTO-MP-AVT-111, 30-1 to 30-22, 2004.

${ }^{14}$ Klein, C., Sachs, W., Henne, U., Engler, R.H., Wiedemann, A., and Konrath, R., "Development of PSP Technique for Application on the VFE-2 65 Delta Wing Configuration in Sub- and Transonic Flow," AIAA Paper 2006-0059, 2006.

${ }^{15}$ Konrath, R., Klein, C., Engler, R. H., and Otter, D., "Analysis of PSP Results Obtained for the VFE-2 $65^{\circ}$ Delta Wing Configuration at Subsonic and Transonic Speed," AIAA Paper 2006-0060, 2006.

${ }^{16}$ Fritz, W., "Numerical Simulation of the Peculiar Subsonic Flow Field about the VFE-2 Delta Wing with Rounded Leading Edge," AIAA Paper 2008-0393, 2008.

${ }^{17}$ Schröder, A., Agocs, J., Frahnert, H., Otter, D., Mattner, H., Kompenhans, J., and Konrath, R., "Application of Stereo-PIV to the VFE-2 $65^{\circ}$ Delta Wing Configuration at Sub- and Transonic Speeds," AIAA Paper 2006-3486, 2006.

${ }^{18}$ Konrath, R., Schröder, A., and Kompenhans, J., "Analysis of PIV Results Obtained for the VFE-2 $65^{\circ}$ Delta Wing Configuration at Sub- and Transonic Speeds," AIAA Paper 2006-3003, 2006.

${ }^{19}$ Konrath, R., Klein, Ch., Schroeder, A., and Kompenhans, J., "Combined Application of Pressure Sensitive Paint and Particle Image Velocimetry to the Flow Above a Delta Wing," 12th International Symposium on Flow Visualization, Sept. 10 14, 2006, DLR Goettingen, Germany, In Grant, I. [Ed.]: CD Rom Proceedings (ISFV12-67.2), Optimage Ltd., Edinburgh, UK, pp. 1 - 14.

${ }^{20}$ Sheplak, M., "The Year in Review: Aerodynamic Measurement Technology," Aerospace America, December 2006, p. 22.

${ }^{21}$ Hummel, D., "The Second International Vortex Flow Experiment (VFE-2): Objectives and First Results," $2^{\text {nd }}$ International Symposium Integrating CFD and Experiments in Aerodynamics, 5 - 6 September 2005, Cranfield University, UK, J. Aerospace Engineering, Vol.. 220, 2006, Nr. 6, pp. 559 - 568.

${ }^{22}$ Hummel, D., "The Second International Vortex Flow Experiment (VFE-2) - Objectives and Present Status," AIAA Paper 2007-4446,2007.

${ }^{23}$ Furman, A. and Breitsamter, Ch., "Delta Wing Steady Pressure Investigations for Sharp and Rounded Leading Edges," In: Rath, H.-J., Holze, C., Heinemann, H.-J., Henke, R. and Hönlinger, H. (Eds), New Results in Numerical and Experimental Fluid Mechanics V, 2004, Vol. 92 of Notes on Numerical Fluid Mechanics and Multidisciplinary Design, pp. 77 - 84.

${ }^{24}$ Furman, A. and Breitsamter, Ch., "Investigations of Flow Phenomena on Generic Delta Wing," ICAS Proceedings Hamburg 2006, Paper 3.1.2 (2006).

${ }^{25}$ Furman, A. and Breitsamter, Ch., "Stereo-PIV and Hot-Wire Investigations on Delta Wing with Sharp and Rounded Leading Edge," ${ }^{\text {st }}$ CEAS Conference Berlin 2007, Paper 2007-430, 2007.

${ }^{26}$ Visbal, M. R., "Computed Unsteady Structure of Spiral Vortex Breakdown on Delta Wings," AIAA Paper 96-2074, 1996.

${ }^{27}$ Müller, J., and Hummel, D., "Numerical Analysis of the Unsteady Flow Above a Slender Delta Wing at Large Angles of Attack," ICAS Proceedings Harrogate 2000, Paper ICA0252, 2000. 